\title{
HIGH-ENERGY EMISSIONS FROM THE GAMMA-RAY BINARY LS 5039
}

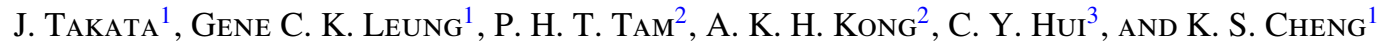 \\ ${ }^{1}$ Department of Physics, University of Hong Kong, Pokfulam Road, Hong Kong \\ 2 Institute of Astronomy and Department of Physics, National Tsing Hua University, Hsinchu, Taiwan; \\ takata@hku.hk,gene930@connect.hku.hk, hrspksc@hku.hk \\ ${ }^{3}$ Department of Astronomy and Space Science, Chungnam National University, Daejeon, Korea \\ Received 2013 December 11; accepted 2014 May 23; published 2014 June 27
}

\begin{abstract}
We study mechanisms of multi-wavelength emissions (X-ray, $\mathrm{GeV}$, and $\mathrm{TeV}$ gamma-rays) from the gamma-ray binary LS 5039. This paper is composed of two parts. In the first part, we report on results of observational analysis using $4 \mathrm{yr}$ data of the Fermi Large Area Telescope. Due to the improvement of instrumental response function and increase of the statistics, the observational uncertainties of the spectrum in the $\sim 100-300 \mathrm{MeV}$ bands and $>10 \mathrm{GeV}$ bands are significantly improved. The present data analysis suggests that the $0.1-100 \mathrm{GeV}$ emissions from LS 5039 contain three different components: (1) the first component contributes to $<1 \mathrm{GeV}$ emissions around superior conjunction, (2) the second component dominates in the $1-10 \mathrm{GeV}$ energy bands, and (3) the third component is compatible with the lower-energy tail of the $\mathrm{TeV}$ emissions. In the second part, we develop an emission model to explain the properties of the phase-resolved emissions in multi-wavelength observations. Assuming that LS 5039 includes a pulsar, we argue that emissions from both the magnetospheric outer gap and the inverse-Compton scattering process of cold-relativistic pulsar wind contribute to the observed $\mathrm{GeV}$ emissions. We assume that the pulsar is wrapped by two kinds of termination shock: Shock-I due to the interaction between the pulsar wind and the stellar wind and Shock-II due to the effect of the orbital motion. We propose that the X-rays are produced by the synchrotron radiation at the Shock-I region and the $\mathrm{TeV}$ gamma-rays are produced by the inverse-Compton scattering process at the Shock-II region.
\end{abstract}

Key words: gamma rays: stars - methods: observational - methods: numerical radiation mechanisms: non-thermal - stars: neutron - X-rays: binaries

\section{INTRODUCTION}

The gamma-ray binary is a class of binary system emitting high-energy ( $\mathrm{GeV}$ and/or $\mathrm{TeV})$ gamma-rays, and comprises a compact object (neutron star or black hole) and a high-mass OB star (see Dubus 2013 for a recent review on the gammaray binaries). Their radiation spectra have a peak in $v F_{\nu}$ around $\mathrm{GeV}$ energy bands and extend up to $1-10 \mathrm{TeV}$ energy bands. Five gamma-ray binaries have been detected so far, namely, the PSR B1259-63/LS2883 system (Aharonian et al. 2005), LS 5039 (Aharonian et al. 2006), LS I+61 303 (Albert et al. 2006), 1FGL J1018.6-5856 (Ackermann et al. 2012), and H.E.S.S. J0632+057 (Hinton et al. 2009). PSR B1259-63/LS 2883 is the only binary system for which the compact object has been confirmed to be a young pulsar.

The GeV gamma-ray observation of the Fermi telescope provides a new challenge for understanding of the non-thermal emission process around gamma-ray binary. The Fermi has revealed that different systems show different properties of the GeV emissions. PSR B1259-63/LS 2883 showed a weak and flare-like emissions during the 2010-2011 periastron passage (Abdo et al. 2011; Tam et al. 2011). The GeV emissions from LS 5039, LS I+61 ${ }^{\circ} 303$, and 1FGL J1018.6-5856 are observed for entire orbit, and the spectra are fitted by a power-law plus exponential cutoff form with a cutoff energy around several $\mathrm{GeV}$. The emissions from LS I+61 303 show a long-term variability related to the 1667 day super-orbital period in the radio band(Ackermann et al. 2013a). No detection of the $\mathrm{GeV}$ emissions has been reported for H.E.S.S. J0632+057.

The gamma-ray binary LS 5039 is known as a relatively compact binary system, for which the separation between two component is $\sim 0.1-0.2 \mathrm{AU}$, and the compact object is moving around an $06.5 \mathrm{~V}$ main-sequence star with a short orbital period
$P_{\mathrm{ob}} \sim 3.9$ days and a moderate eccentricity $(e \sim 0.24-0.35$; Casares et al. 2005; Aragona et al. 2009; Sarty et al. 2011). The binary system is a source of non-thermal emission in radio (Moldón et al. 2012), X-ray (Takahashi et al. 2009), and gammaray (Abdo et al. 2009 for GeV; Aharonian et al. 2006 for $\mathrm{TeV}$ ) bands and exhibits temporal variations in its emission and spectrum.

The modulating GeV emission from LS 5039 has been confirmed by the Fermi-Large Area Telescope (LAT; Abdo et al. 2009). The pattern of the orbital modulation of $\mathrm{GeV}$ emissions shows in anti-phase with $\mathrm{X}$-ray and $\mathrm{TeV}$ gammaray emissions (see Figure 12); the observed $\mathrm{GeV}$ flux (or $\mathrm{X} / \mathrm{TeV}$ fluxes) becomes maximum around the superior conjunction (or inferior conjunction) and becomes minimum around the inferior conjunction (or superior conjunction). The spectrum in $0.1-10 \mathrm{GeV}$ bands is harder when the emission is weaker. The phase-averaged spectrum shows a cutoff around $\sim 2 \mathrm{GeV}$, but Hadasch et al. (2012) found an emission feature above $10 \mathrm{GeV}$, which will be compatible with the lower-energy tail of the $\mathrm{TeV}$ emissions.

The origin of the $\mathrm{GeV}$ emissions from the LS 5039 remains to be solved. Because the spectral shape of LS5039 measured by Fermi resembles those of the gamma-ray-emitting pulsars, it has been suggested that LS 5039 includes a young pulsar and the emissions from the magnetosphere or the cold-relativistic pulsar wind produce the $\mathrm{GeV}$ emissions (Sierpowska-Bartosik \& Torres 2007; Kapala et al. 2010; Torres 2011). On the other hand, the inverse-Compton (IC) scattering process of the pulsar wind accelerated by the inter-binary shock was also proposed to explain the GeV emissions (Yamaguchi \& Takahara 2012; Zabalza et al. 2013).

The main purposes of this study are (1) to present results of the observational analysis using 4 yr Fermi data, which provide 
us with more detailed information on the $\mathrm{GeV}$ emissions from LS 5039, and (2) to develop a model to discuss the emission processes of the $\mathrm{X}$-ray, $\mathrm{GeV}$, and $\mathrm{TeV}$ gamma-rays. The present paper is composed of two parts. In the first part, we report on results of the $4 \mathrm{yr}$ observations of Fermi. Although Hadasch et al. (2012) found the emissions above $10 \mathrm{GeV}$ with $2.5 \mathrm{yr}$ Fermi data, the large uncertainty in the phase-resolved spectra in those energies prevents us from understanding the detailed spectral behavior above $10 \mathrm{GeV}$. Furthermore, the emissions around $100 \mathrm{MeV}$ are strongly affected by the background model. Therefore, in this paper, we perform a more detailed analysis with updated instrument response functions to obtain a more complete understanding of the spectral behavior in $100 \mathrm{MeV}$ and $>10 \mathrm{GeV}$ energy bands.

In the second part, we develop the emission model in which the emissions from the magnetospheric outer gap and from cold-relativistic pulsar wind contribute to the $\mathrm{GeV}$ emissions of LS 5039 and compare the predicted emission properties with the results of Fermi observation. We also study the $\mathrm{X}$-ray and $\mathrm{TeV}$ gamma-ray emissions from the intra-binary shock and discuss the properties of the phase-resolved spectra in the multi-wavelength bands (X-ray, GeV, and TeV). In Section 2, we report on the results of our analysis of the $4 \mathrm{yr}$ Fermi data. We describe the emission model in Section 3 and compare the model predictions with the results of the multi-wavelength observations in Section 4. A discussion and a brief summary are given in Sections 5 and 6, respectively.

\section{DATA ANALYSIS AND RESULTS OF THE Fermi DATA}

\subsection{Data Set}

In this study, we used data collected starting 2008 August 14 and extending until 2012 May 19. The observation time was limited by the availability of the timing model of the nearby gamma-ray pulsar PSR J1826-1256, which was needed for removing the contribution of the pulsar. The timing model was adopted from the Fermi LAT Multiwavelength Coordinating Group $^{4}$ (Ray et al. 2011). The data were reduced and analyzed using the Fermi Science Tools package (v9r32p5), available from the Fermi Science Support Center. ${ }^{5}$ We selected only events in the Reprocessed Pass 7 "Source" class and used the P7REP_SOURCE_V15 version of the instrumental response functions. To reduce contamination from the Earth's albedo, we excluded time intervals when the region of interest (ROI) was observed at zenith angles greater than $100^{\circ}$ or when the rocking angle of the LAT was greater than $52^{\circ}$. To minimize background from the nearby gamma-ray pulsar PSR J1826-1256, we excluded events arriving in the pulse phase intervals $0.05-0.2$ and $0.6-0.75$ of the pulsar.

\subsection{Spectral Analysis}

The gtlike tool was used for spectral analysis. We used photons between 0.1 and $300 \mathrm{GeV}$ within a $20^{\circ} \times 20^{\circ} \mathrm{ROI}$ centered at the position of LS 5039. For source modeling, all 2FGL catalog sources (Nolan et al. 2012) within $19^{\circ}$ of the ROI center, the galactic diffuse emission (gll_iem_v05.fit), and isotropic diffuse emission (iso_source_v05.txt) were included.

\footnotetext{
4 https://confluence.slac.stanford.edu/display/GLAMCOG/LAT+Gammaray+Pulsar+Timing+Models

5 http://fermi.gsfc.nasa.gov/ssc/data/analysis/software/
}

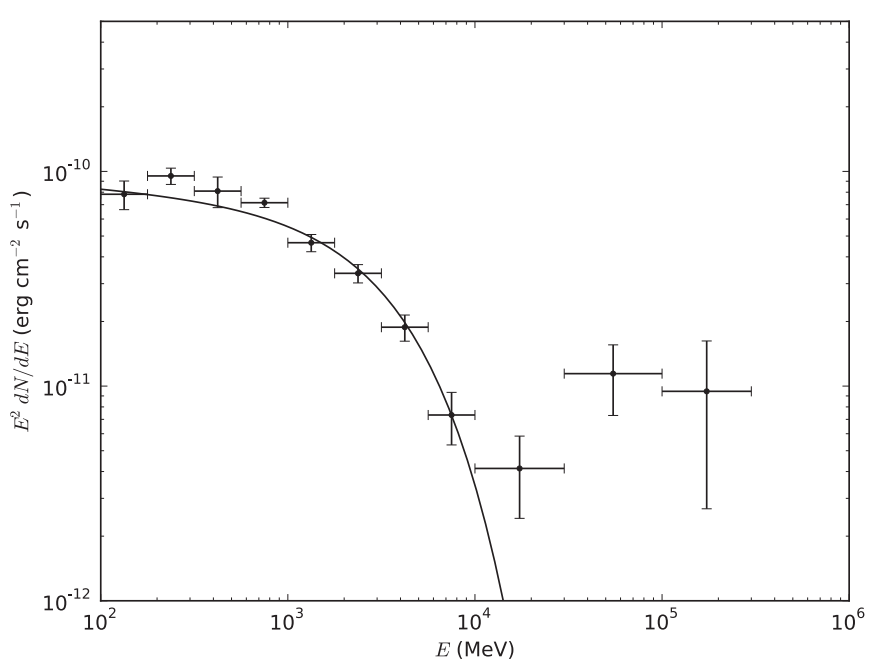

Figure 1. Phase-averaged spectrum of LS 5039. The solid line shows the best-fit model for the full energy band.

\subsubsection{Phase-averaged Spectrum}

We modeled LS 5039 with a power law with an exponential cutoff:

$$
\frac{d N}{d E}=N_{0}\left(\frac{E}{E_{0}}\right)^{-\Gamma} \exp \left(-\frac{E}{E_{\text {cutoff }}}\right) .
$$

The spectral types of other point sources in 2FGL are modeled according to the spectral types in the catalog, with the spectral parameters of sources more than $10^{\circ}$ away from the ROI center fixed to the catalog values. The best-fit parameters are $\Gamma=2.06 \pm 0.02_{\text {stat }}$ and $E_{\text {cutoff }}=3.42 \pm 0.17_{\text {stat }} \mathrm{GeV}$.

Spectral points were obtained by performing a fit in each energy band, fixing the spectral parameters of sources more than $4^{\circ}$ away from the ROI center, leaving the flux normalization constants of all other sources and the diffuse background free. The sources that were left free were modeled by power laws. In addition, an initial fit was performed and sources with TS $<$ 0 in that energy band were removed. Figure 1 shows the phaseaveraged spectrum. Significant emission is observed at $E>$ $10 \mathrm{GeV}$, in agreement with Hadasch et al. (2012) and Ackermann et al. (2013b).

\subsubsection{Phase-resolved Spectra}

We first performed phase-resolved analysis following the H.E.S.S. analysis by Aharonian et al. (2006). We set phase zero at the periastron $(\phi=0$ with MJD $=51,942.59)$ and divided one orbit into two phases, that is, the superior conjunction phase (SUPC phase; $0<\phi<0.45$ and $0.9<\phi<1$ ), which includes SUPC $(\phi=0.06)$, and the inferior conjunction phase (INFC phase, $0.45<\phi<0.9)$, which includes INFC ( $\phi=0.72)$. We performed similar likelihood analysis in the two phase intervals. In the fitting, all of the spectral parameters of LS 5039 were left free, while other spectral parameters were fixed to the phaseaveraged values, except for the flux normalization parameters of sources within $5^{\circ}$ of the ROI center and the galactic and isotropic diffuse emissions. The best-fit parameters for the SUPC (INFC) are $\Gamma=1.96 \pm 0.09_{\text {stat }}\left(1.89 \pm 0.12_{\text {stat }}\right)$ and $E_{\text {cutoff }}=2.86 \pm 0.57_{\text {stat }}\left(3.58 \pm 0.97_{\text {stat }}\right) \mathrm{GeV}$. The spectrum is shown in Figure 2. It is found that the flux in $\sim 100-300 \mathrm{MeV}$ has noticeably decreased compared with previous studies done by Abdo et al. (2009) and Hadasch et al. (2012). This may be due to the improvement in instrumental response functions and data from Pass 6 to Reprocessed Pass 7. The flux beyond $\sim 10 \mathrm{GeV}$ 

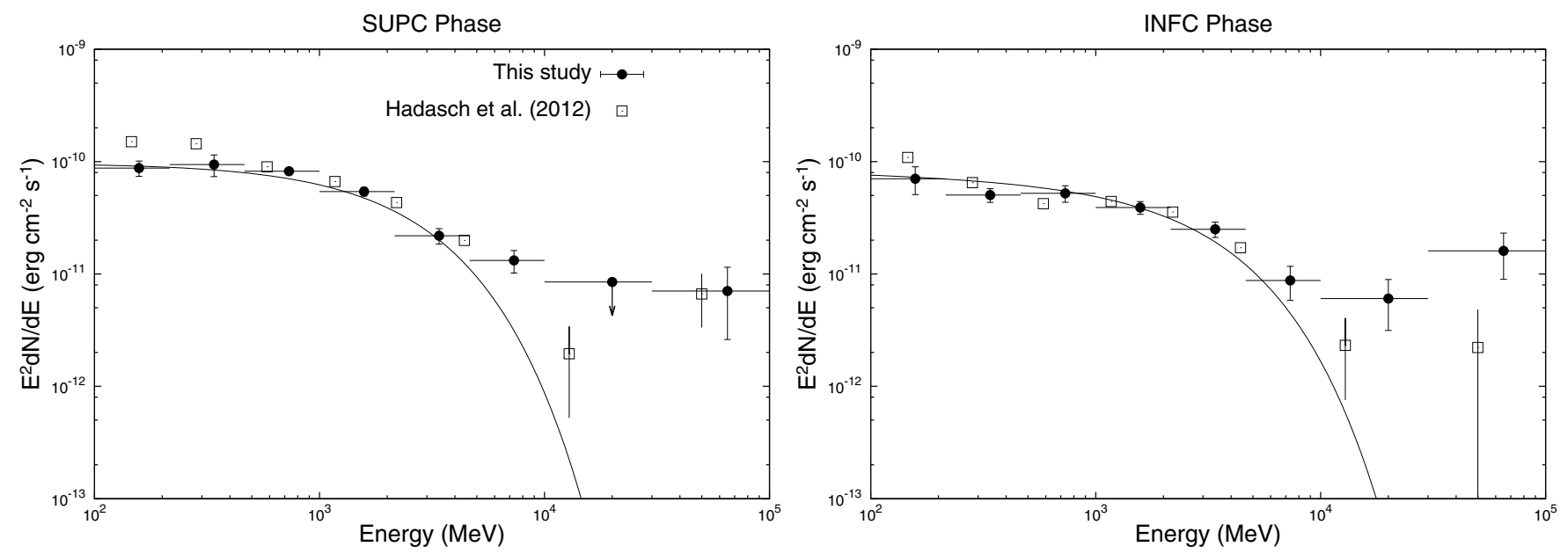

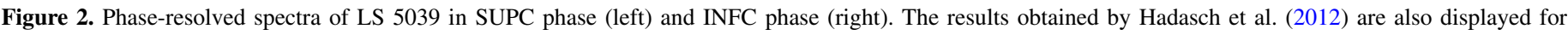
comparison. The solid lines show the bet-fitting functions described in Section 2.2.2.

is also increased compared with the previous studies, which is also seen in other results with the Reprocessed Pass 7 data (e.g., see Bregeon et al. 2013).

Due to the increased statistics in this study, we are allowed to divide the observation time into more orbital phase bins. We divided the observation time into three equally spaced orbital phase bins: $[0.17,0.5]$ (bin 1), [0.5, 0.83] (bin 2, INFC), and $[0.83,1] \cup[0,0.17]$ (bin 3, SUPC). Both the first and second intervals touch the apastron, but only the second interval contains the INFC. This cut is chosen to better isolate the INFC from the apastron. In addition, the first and second phase bins exclude the SUPC and the third phase bin includes the emissions at the SUPC. The results are shown in Figure 3 for comparing with results of the theoretical model discussed in Section 3. The figure shows that the spectra of two orbital bins excluding the superior conjunction (upper panels) have a clear spectral cutoff at $\sim 2 \mathrm{GeV}$, and the spectra below $10 \mathrm{GeV}$ resemble each other. At the phase bin containing the SUPC (lower left panel), an enhancement at $0.1-0.3 \mathrm{GeV}$ is exclusively seen, and the spectrum below $10 \mathrm{GeV}$ is softer than the other two phase bins. This suggests that the emissions below $10 \mathrm{GeV}$ are composed of the two components, that is, one contributes to emissions around the SUPC in $<1 \mathrm{GeV}$ bands and the other dominates in the 1-10 $\mathrm{GeV}$ emissions for the entire orbit. As shown in Figure 3, the change of the spectral slope at around $20 \mathrm{GeV}$ in each phase bin suggests existence of an additional component that is compatible with the lower-energy tail of the $\mathrm{TeV}$ emissions

\subsection{Orbital Light Curves}

To obtain light curves, we performed likelihood analysis similar to that in Section 2.2.2. The orbital modulation for the flux is summarized in Figure 4, in which the left and right panels display the light curves in two energy bands, 0.2-300 $\mathrm{GeV}$ and 1-100 $\mathrm{GeV}$, respectively. In Figure 4, results of the theoretical model are also displayed for comparison. The observed trends of flux modulation of the two energy bands are similar, but the amplitudes in the modulations are significantly different. The flux including lower-energy photons (0.2-300 $\mathrm{GeV})$ is modulated by a factor of $\sim 5$ while that in the highenergy band $(1-100 \mathrm{GeV})$ is modulated by only a factor of $\sim 2$. This indicates that the variation of spectral hardness along the orbital phase is mainly due to the modulation in the low-energy band rather than the high-energy band, and the high-energy bands are dominated by a component which does not vary with the orbital phase. This could also support the hypothesis that the $\mathrm{GeV}$ emissions from LS 5039 are composed of several components with different characteristic energies. This feature is more clearly seen in our results than the results in previous studies. To qualitatively describe the evolution of the hardness of the emissions, we extracted the photon index of each phase interval by assuming a simple power law in $0.2-300$ energy bands,

$$
\frac{d N}{d E}=N_{0}\left(\frac{E}{E_{0}}\right)^{-\Gamma} .
$$

It is clear from Figure 1 that there is a curvature in the spectra, and a single power-law function would not be appropriate at some orbital phases. However, because of the reduced statistics in individual phase bins, it is not possible to distinguish a simple power law from a power law with an exponential cutoff with statistical significance in some bins. A fit with a simple power law can provide a quantitative indicator of the hardness of the spectrum. The result is shown in Figure 3 (bottom right panel). The spectrum is the softest around SUPC and hardest in a broad region centered around the apastron $(\phi=0.5)$.

In summary, the results of the $4 \mathrm{yr}$ observations by Fermi improve our understanding of the GeV emissions from LS 5039. The current results suggest that $0.1-100 \mathrm{GeV}$ emissions from LS 5039 are likely composed of three different components: (1) the first component contributes to $<1 \mathrm{GeV}$ emissions around superior conjunction, (2) the second component dominates in 1-10 GeV energy bands for the entire orbit, and (3) the third component is compatible with the lower-energy tail of the TeV emissions.

\section{THEORETICAL MODEL}

In the last section, we discussed the evidence of two components of the emissions in the $0.1-10 \mathrm{GeV}$ bands observed by Fermi. In this paper, we propose that the emissions in the 1-10 GeV energy band are dominated by the magnetospheric emissions, while $\sim 0.1 \mathrm{GeV}$ gamma-rays around the superior conjunction are mainly produced by the emissions from the cold-relativistic pulsar wind. Hence, our model predicts that the emissions in 1-10 GeV energy band are pulsed.

Figure 5 shows the schematic view of the LS 5039 system discussed in this paper. Our model assumes that LS 5039 includes 

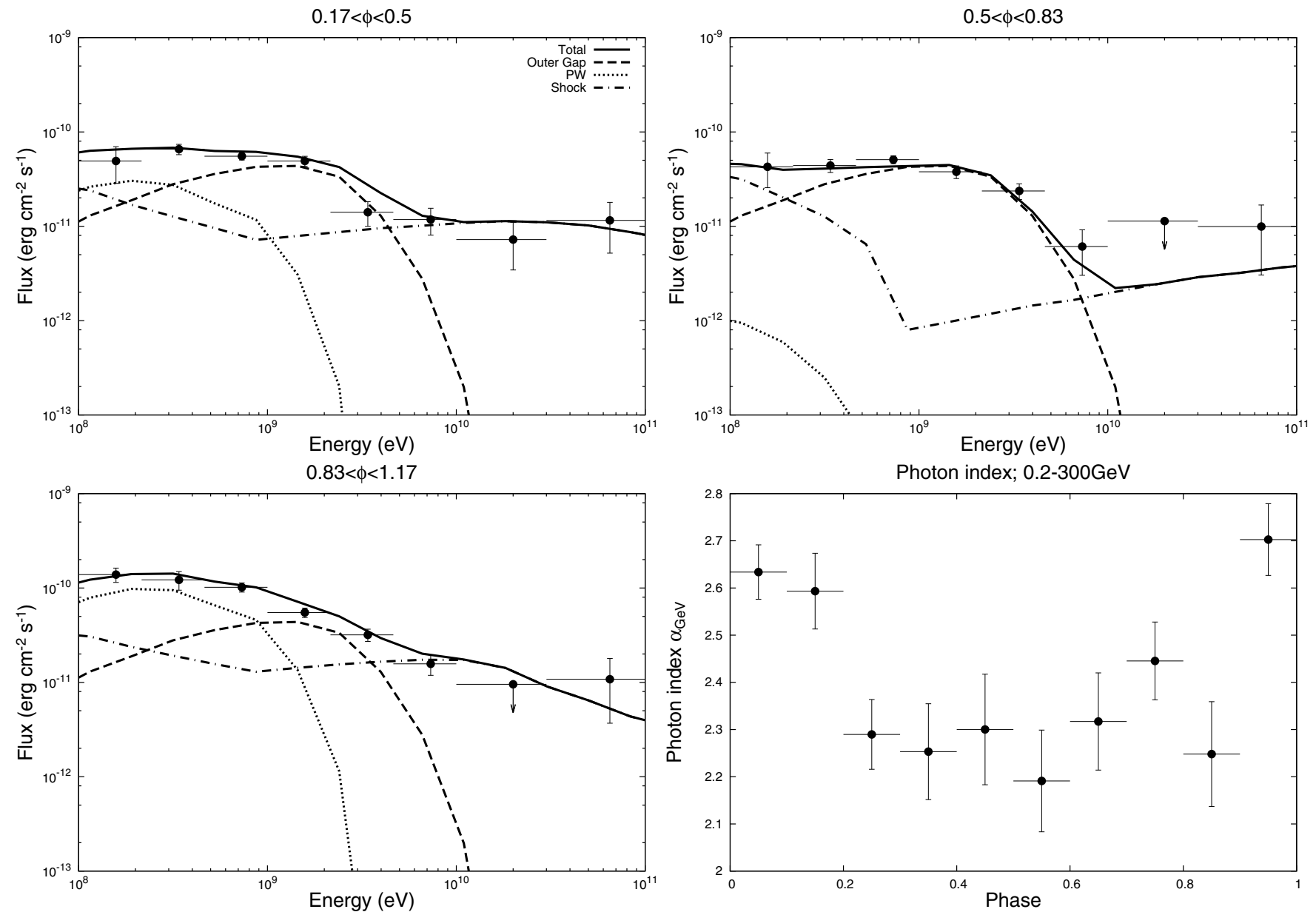

Figure 3. Phase-resolved spectra and the photon index of GeV gamma-ray emissions from LS 5039. The top left, top right, and bottom left panels show the spectra for the orbital interval $0.17<\phi<0.5,0.5<\phi<0.83$, and $0.83<\phi<0.17$, respectively. The bottom right panel shows the photon index fitted by a single power-law function on $0.2-300 \mathrm{GeV}$. The results of current Fermi data analysis are represented by the filled circles. The solid lines show the results of the theoretical model (Section 3), which includes emissions from the outer gap (dashed lines), the cold-relativistic pulsar wind (dotted lines), and the shocked pulsar wind (dashed-dotted lines).
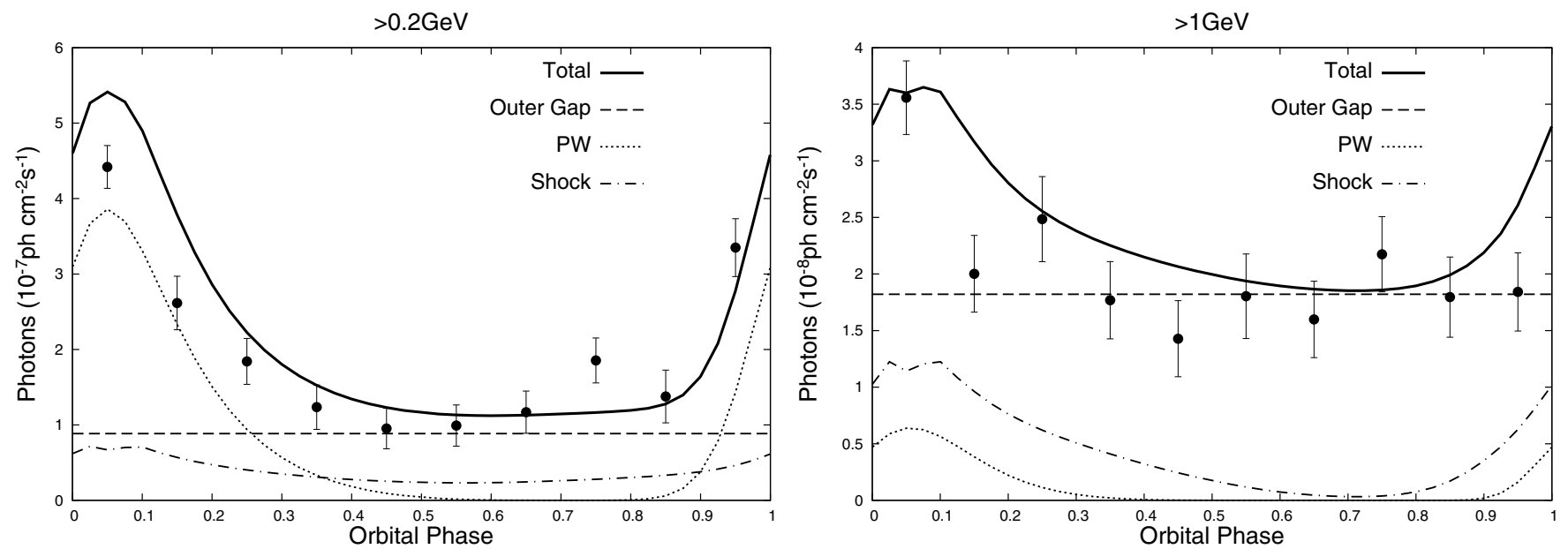

Figure 4. Variation of the integrated flux in $>0.2 \mathrm{GeV}$ bands (left panel) and $>1 \mathrm{GeV}$ bands (right panel). The symbols and the lines correspond to the same case as Figure 3.

a pulsar. The pulsar is wrapped by the termination shocks (Section 3.1), where the pulsar wind is stopped. The emissions from the magnetosphere (Section 3.2) and the IC scattering process of the cold-relativistic pulsar wind (Section 3.3) contribute to the observed $\mathrm{GeV}$ emissions. The synchrotron radiation and the IC process of the shocked pulsar wind produce the X-rays and $\mathrm{TeV}$ gamma-rays, respectively (Section 3.4).

We note that the shock geometry applied in this study (see Section 3.1) resembles one presented in Zabalza et al. (2013), who applied two shock regions: the first shock (Shock-I) is 


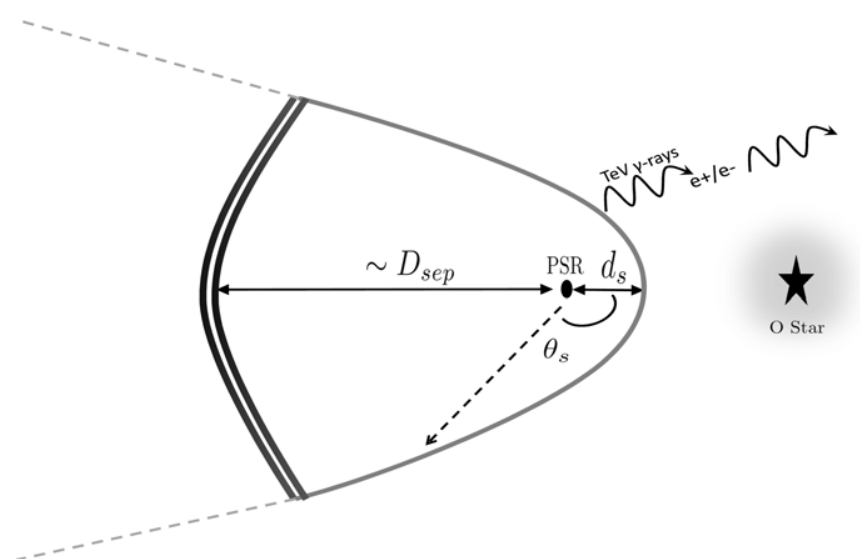

Figure 5. Schematic view of proposed picture of LS 5039 system. LS 5039 comprises a pulsar and an $\mathrm{O}$ star, and the interaction between the pulsar wind and the stellar wind from the companion star forms a termination shock (Shock-I, thick solid line). The distance to the shock apex $\left(d_{s}\right)$ from the pulsar is determined by the balance between the pulsar wind pressure and stellar wind pressure. The effect of the orbital motion of the pulsar also produces a termination shock in the opposite direction of the companion star (Shock-II, double solid lines). The distance to shock from the pulsar is of the order of the orbital separation $\left(D_{\text {sep }}\right)$, for which the ram pressure of the stellar wind due to Coriolis force and the ram pressure of the pulsar are in balance. The $\mathrm{GeV}$ emissions are mainly produced by the curvature radiation in the magnetospheric outer gap and by the inverse-Compton scattering of the cold-relativistic pulsar wind. The synchrotron process and inverse-Compton process of the shocked pulsar wind produce the $\mathrm{X}$-rays and $\mathrm{TeV}$ gamma-rays, respectively. The TeV gamma-rays emitted from the shocked pulsar wind create new electrons and positron pairs, and they initiate the pair-creation cascade, if they propagate toward the companion star.

located between the pulsar and the companion star, and the second shock (Shock-II) is in the opposite direction from the companion star. Both our model and Zabalza et al. (2013) assume that $\mathrm{TeV}$ gamma-rays are mainly produced by the Shock-II region (see Section 4.2). The main difference between us and Zabalza et al. (2013) is our X-ray/GeV emission processes. Our model predicts that the particles accelerated at the ShockI produce the X-rays via synchrotron radiation, while Zabalza et al. (2013) proposed that the particles accelerated at Shock-I produce the Gev emissions via the IC process. For emissions in $0.1-10 \mathrm{GeV}$ bands, we expect that the emissions are mainly produced by the magnetospheric particles in the outer gap and the cold-relativistic pulsar wind. Since Zabalza et al. (2013) mainly focused on the $\mathrm{GeV} / \mathrm{TeV}$ gamma-ray emission process and had difficulty explaining the X-ray emission process, we develop an emission model that covers X-ray to TeV energy bands.

\subsection{Shock Geometry}

We assume that two kinds of termination shock exist around the pulsar (see Figure 5). First, balancing between the pressures of the pulsar wind and the stellar wind produces a cone-shape shock between the pulsar and the companion star (Shock-I). The opening angle and the geometry of the cone-shape shock are determined by the ratio of the momenta of the two winds (e.g., Eichler \& Usov 1993; Canto et al. 1996),

$$
\eta \equiv \frac{L_{\mathrm{sd}}}{\dot{M} v_{w} c},
$$

where $L_{\mathrm{sd}}$ is the spin-down energy, $\dot{M}$ is the mass-loss rate of the outflow from the companion star, and $v_{w}$ is the velocity of the outflow. The distance to the shock apex from the pulsar can be determined by $r_{\text {apex }}=D_{\text {sep }} \eta^{1 / 2} /\left(1+\eta^{1 / 2}\right)$, where $D_{\text {sep }}$ is the separation between two stars. In this paper, we apply

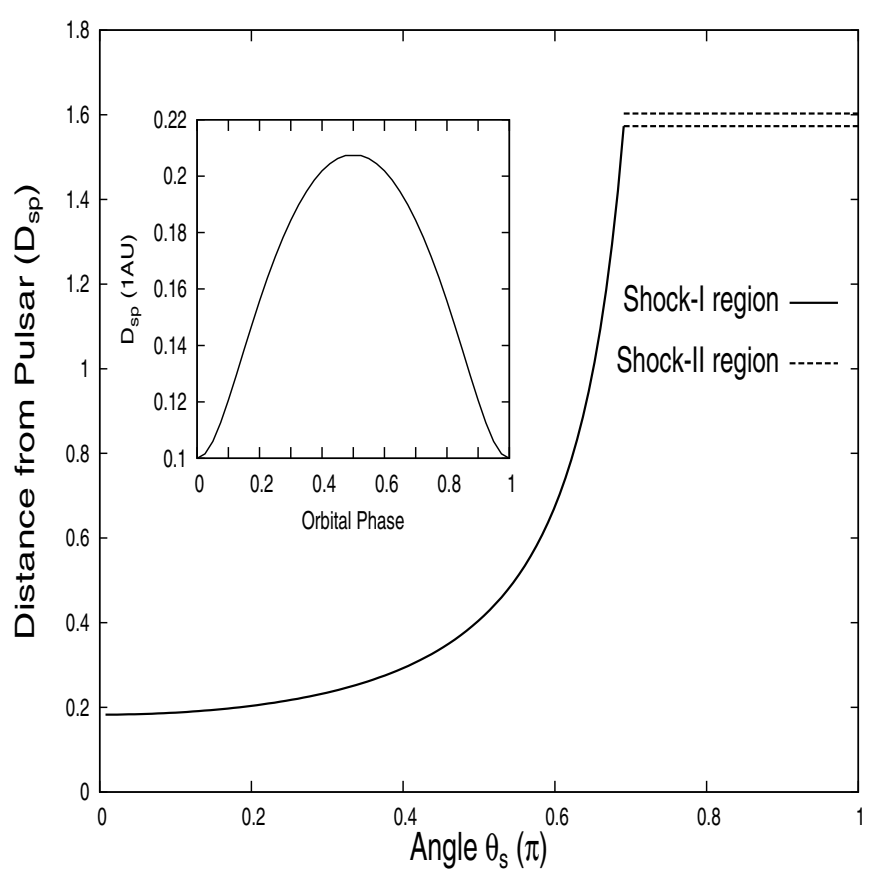

Figure 6. Distance to termination shock from the pulsar with respect to the angle measured $\left(\theta_{s}\right.$; see Figure 5$)$ from the direction of the companion star. Solid line: distance to the shock caused by the interaction between the pulsar wind and the stellar wind (Shock-I). The result is for the momentum ration of $\eta=0.05$. Double solid line: distance to the shock caused by the effect of the orbital motion of the pulsar (Shock-II). In the calculation, we assume $r_{s}=1.5 D_{\text {sep }}$. The subpanel in the figure shows the separation between two stars as a function of the orbital phase.

$\eta=0.05$ with $L_{\text {sd }} \sim 2 \times 10^{36} \mathrm{erg} \mathrm{s}^{-1}, \dot{M} \sim 10^{-7} M_{\odot} \mathrm{yr}^{-1}$, and $v_{w} \sim 2 \times 10^{8} \mathrm{~cm} \mathrm{~s}^{-1}$.

Second, recent results of two dimensional (2D) hydrodynamic simulation of LS 5039 (e.g., Bosch-Ramon et al. 2012) have suggested that the effect of the orbital motion also produces a pulsar wind termination shock even in the opposite direction from the companion star (Shock-II; see the double solid line in Figure 5). The distance from the pulsar is found to be of the order of the orbital separation, for which the ram pressure of the stellar wind due to Coriolis force and the ram pressure of the pulsar are in balance. Zabalza et al. (2013) provided an approximate expression for location of the shock as

$$
x_{\mathrm{cor}} \sim \sqrt{\frac{L_{\mathrm{sd}} v_{w}}{\dot{M} c\left(2 \Omega_{o}\right)^{2}}},
$$

where $\Omega_{o}$ is the angular velocity of the pulsar around the star. With $\Omega_{o} \sim 10^{-5} \mathrm{~s}^{-1}$, the expression (4) yields $x_{\text {cor }} \sim$ $0.15 \mathrm{AU} \sim 1-2 \mathrm{D}_{\text {sep. }}$. In this paper, we assume $r_{s}=1.5 D_{\text {sep }}$ as the radial distance to the Shock-II from the pulsar. Figure 6 shows the distance to the shock as a function of angle measured from the direction of the companion star.

It has been pointed out that three-dimensional (3D) simulation will give a more complex structure of Shock-II because the instability will develop faster and more disruptive in 3D simulation than 2D (Bosch-Ramon et al. 2012). Because the detailed structure of the shock-II region given by the 3D simulation has not been known for LS 5039 system, we apply the result of 2D calculation in this study.

\subsection{Magnetospheric Emission}

We calculate the expected spectra of the gamma-ray emissions from the outer gap accelerator in the pulsar magnetosphere 
(Cheng et al. 1986). In the outer gap, the electrons and positrons are accelerated by the electric field along the magnetic field lines. The accelerated particles emit the GeV gamma-rays through the curvature radiation process. The typical magnitude of the accelerating electric field is

$$
E_{\|} \sim \frac{f_{\mathrm{gap}}^{2} B\left(R_{\mathrm{lc}}\right) R_{\mathrm{lc}}}{R_{c}}
$$

where $B$ is the local magnetic field strength, $R_{\mathrm{lc}}=c / \Omega$ is the radius of the light cylinder, $R_{c}$ is curvature radius of the magnetic field line, and $f_{\text {gap }}$ is the ratio of the gap thickness in the trans-field direction and the light cylinder radius at the light cylinder. The accelerated electrons and positrons produce gamma-rays via the curvature radiation process. The Lorentz factor of the electrons/positrons is estimated by balancing between the acceleration force and back reaction force of the curvature radiation,

$$
\begin{aligned}
\Gamma_{e} \sim & \left(\frac{3 R_{c}^{2}}{2 e} E_{\mathrm{ll}}\right)^{1 / 4} \sim 2 \times 10^{7}\left(\frac{f_{\mathrm{gap}}}{0.1}\right)^{1 / 2}\left(\frac{\Omega}{10^{2} \mathrm{~s}^{-1}}\right)^{1 / 2} \\
& \times\left(\frac{B\left(R_{\mathrm{lc}}\right)}{10^{5} \mathrm{G}}\right)^{1 / 4}\left(\frac{R_{c}}{R_{\mathrm{lc}}}\right)^{1 / 4} .
\end{aligned}
$$

Typical energy of the curvature radiation is found to be of the order of $\sim \mathrm{GeV}$,

$$
E_{c} \sim \frac{3 h c \Gamma_{e}^{3}}{4 \pi R_{c}} \sim 800\left(\frac{\Gamma_{e}}{2 \times 10^{7}}\right)^{3}\left(\frac{\Omega}{10^{2} \mathrm{~s}^{-1}}\right)\left(\frac{R_{c}}{R_{\mathrm{lc}}}\right)^{-1} \mathrm{MeV}
$$

The luminosity of the gamma-ray emissions from the outer gap becomes

$$
L \gamma \sim f_{\text {gap }}^{3} L_{\text {sd }} \sim 3.8 \times 10^{35} f_{\text {gap }}^{3}\left(\frac{P}{0.1 \mathrm{~s}}\right)^{-4}\left(\frac{B_{s}}{10^{12} \mathrm{G}}\right)^{2} \mathrm{ergs}^{-1},
$$

where $L_{\mathrm{sd}}=2(2 \pi)^{4} B_{s}^{2} R_{s}^{6} /\left(3 c^{3} P^{4}\right)$ is the spin-down power, $P$ is the pulsar's spin period, $B_{s}$ is the stellar magnetic field, and $R_{s}=10^{6} \mathrm{~cm}$ is the radius of neutron star. The fractional gap thickness is estimated as

$$
f_{\text {gap }}=\min \left(f_{m}, f_{p}\right)
$$

where $f_{m}=0.25 K(P / 0.1 \mathrm{~s})^{1 / 2}$ with $K \sim 2$ and $f_{p}=$ $5.5 P^{26 / 21}\left(B_{s} / 10^{12} \mathrm{G}\right)^{-4 / 7}$ (Takata et al. 2010). A more detailed description of the outer gap model can be found in Wang et al. (2010).

\subsection{Inverse-Compton Emission from Cold-relativistic Pulsar Wind}

It is possible that the IC scattering of cold-relativistic pulsar wind off the stellar photons produces high-energy gamma-rays (Ball \& Kirk 2000; Khangulyan et al. 2011, 2012). For the LS 5039 system, this IC component could play an important role in explaining the observed emissions. As we show in Equation (12), the typical Lorentz factor of the cold-relativistic pulsar wind is $\Gamma_{0} \sim 10^{4}$. Hence, the IC scattering process occurs in the Thomson regime. With typical separation of two stars $\left(D_{\text {sep }} \sim 0.1 \mathrm{AU}\right)$, the optical depth of IC process will be of the order of unity (by ignoring the effect of the collision angle):

$$
\begin{aligned}
\tau_{\mathrm{IC}}\left(D_{\mathrm{sep}}=\right. & 0.1 \mathrm{AU}) \sim n_{\mathrm{ph}} \sigma_{\mathrm{IC}} r_{s} \sim 5\left(\frac{\sigma_{\mathrm{IC}}}{\sigma_{T}}\right)\left(\frac{T_{\mathrm{eff}}}{3 \times 10^{4} \mathrm{~K}}\right)^{3} \\
& \times\left(\frac{R_{*}}{9 R_{\odot}}\right)^{2}\left(\frac{D_{\mathrm{sep}}}{0.1 \mathrm{AU}}\right)^{-2}\left(\frac{r_{s}}{0.01 \mathrm{AU}}\right)
\end{aligned}
$$

where $r_{s}$ is the distance to the shock, $T_{\text {eff }}$ is the effective temperature of the companion, and $\sigma_{T}$ is the Thomson crosssection. The luminosity becomes of the order of

$$
L_{\mathrm{IC}} \sim \frac{\Gamma_{0} k T_{\mathrm{eff}}}{m_{e} c^{2}} L_{\mathrm{sd}} \sim 0.03\left(\frac{k T_{\mathrm{eff}}}{3 \mathrm{eV}}\right)\left(\frac{\Gamma_{0}}{5 \times 10^{3}}\right) L_{\mathrm{sd}} .
$$

Hence, the IC process of the cold-relativistic pulsar wind will produce observable high-energy gamma-rays.

The characteristic energy of the IC photons depends on the Lorentz factor of the cold-relativistic pulsar wind. If the pulsar wind is a kinetically dominated flow, the typical Lorentz factor of the bulk flow will be

$$
\Gamma_{W, 0} \sim \Gamma_{W, L} \sigma_{L} \sim 10^{4}\left(\frac{\kappa}{10^{5}}\right)^{-1}\left(\frac{L_{\mathrm{sd}}}{10^{36} \mathrm{erg} \mathrm{s}^{-1}}\right)^{1 / 2},
$$

where $\Gamma_{W, L}$ and $\sigma_{L}=B^{2}\left(R_{\mathrm{lc}}\right) /\left[4 \pi \Gamma_{W, L} \kappa m_{e} c^{2} n_{\mathrm{GJ}}\left(R_{\mathrm{lc}}\right)\right]$ are the Lorentz factor and the magnetization parameter of the pulsar wind at the light cylinder, respectively. In addition, $n_{\mathrm{GJ}}\left(R_{\mathrm{lc}}\right)=\Omega B\left(R_{\mathrm{lc}}\right) /(2 \pi c e)$ is the Goldreich-Julian number density and $\kappa$ is the multiplicity. The observed power of the synchrotron nebulae around the pulsars implies a multiplicity of $\kappa=10^{4-5}$ (De Jager et al. 1996; De Jager 2007; Harding \& Muslimov 2011). The radiation per unit energy power unit solid angle of single particle with a Lorentz factor $\Gamma_{W}$ is given by

$$
\frac{d P_{\mathrm{IC}}}{d \Omega_{1}}=\mathcal{D}^{2} \int_{0}^{\theta_{c}}\left(1-\beta \cos \theta_{0}\right) I_{b} / h \frac{d \sigma^{\prime}}{d \Omega^{\prime}} d \Omega_{0},
$$

where $d \sigma^{\prime} / d \Omega^{\prime}$ is the differential Klein-Nishina cross-section, $\mathcal{D}=\Gamma_{W}^{-1}\left(1-\beta \cos \theta_{1}\right)^{-1}, \theta_{1}$, and $\theta_{0}$ describe the angle between the direction of the particle motion and the propagating direction of the scattered photons and background photons, respectively. In addition, $I_{b}$ is the stellar photon field and $\theta_{c}=\sin ^{-1} R_{*} / r$ expresses the angular size of the star as seen from point $r$. For the target stellar photon field, we take the stellar radius $R_{*}=9 R_{\odot}$, where $R_{\odot}$ is the solar radius, and an effective temperature $k T_{\text {eff }}=3.4 \mathrm{eV}$. With $k T_{\text {eff }}=3.4 \mathrm{eV}$, the scattering process of the electrons with a Lorentz factor of $\gamma_{e} \geqslant 2 \times 10^{5}$ occurs in the Klein-Nishina regime (see Figure 9), implying the existence of a break in the IC spectrum at $\sim 10^{11} \mathrm{eV}$.

A mono-energetic assumption for the distribution of electrons and positrons in the pulsar wind had been assumed as a first approach to the problem (e.g., Takata \& Taam 2009). It has been suggested however that the energy distribution as a result of dissipation of the magnetic energy to the particle energy can be different from the mono-energetic distribution (SierpowskaBartosik \& Torres 2008 and references therein). In this paper, we explore the emissions with a relativistic Maxwell distribution of the form

$$
f\left(r, \Gamma_{W}\right)=K(r) \Gamma_{W}^{2} \exp \left(-\frac{3 \Gamma_{W}}{\Gamma_{0}(r)}\right),
$$

which provides the averaged Lorentz factor of

$$
\left\langle\Gamma_{W}(r)\right\rangle \equiv \frac{\int_{1}^{\infty} \Gamma_{W} f\left(r, \Gamma_{W}\right) d \Gamma_{W}}{\int_{1}^{\infty} f\left(r, \Gamma_{W}\right) d \Gamma_{W}} \sim \Gamma_{0}(r) .
$$




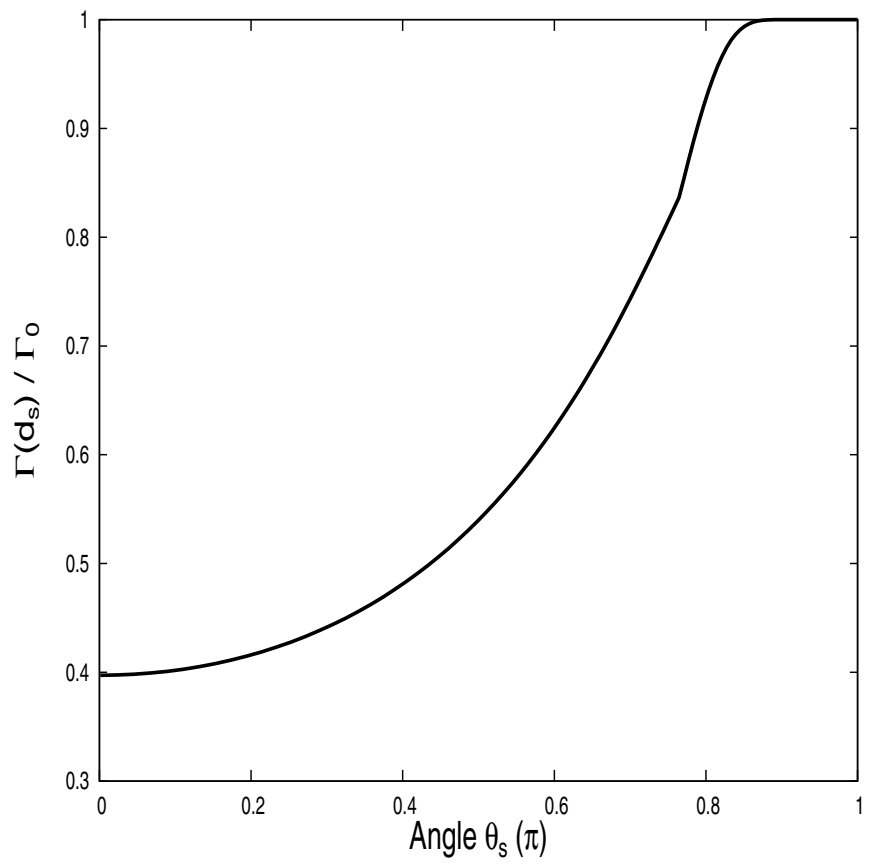

Figure 7. Ratio of Lorentz factor at the shock and initial Lorentz factor of the cold-relativistic pulsar wind with respect to the angle measured from the direction of the companion star. The cold-relativistic pulsar wind loses the energy via the inverse-Compton scattering off the stellar photons.

We assume that the distance $\left(r_{i}\right)$ from the pulsar at which the kinetically dominated pulsar wind is formed is smaller than the shock distance and that the averaged Lorentz factor at $r=r_{i}$ is $\Gamma_{0}\left(r_{i}\right)=5 \times 10^{3}$. The normalization $K\left(r_{i}\right)$ at $r=r_{i}$ is calculated from

$$
m_{e} c^{2} \int_{1}^{\infty} \Gamma_{W} f\left(r_{i}, \Gamma_{W}\right) d \Gamma_{W}=\frac{L_{\mathrm{sd}}}{4 \pi r_{i}^{2} c} .
$$

The Lorentz factor of the pulsar wind evolves with the distance due to the energy loss from the IC scattering process. For example, Figure 7 shows the Lorentz factor of the pulsar wind at the shock as a function of the angle measured from the direction of the companion star, where the initial Lorentz factor is $\Gamma_{0}=5 \times 10^{3}$. Figure 7 shows that for the cold-relativistic pulsar wind propagating toward the companion star $\left(\theta_{s} \sim 0\right)$, $40 \%-50 \%$ of the initial energy is released before the shock, while for the pulsar wind propagating in opposite direction of the companion star $\left(\theta_{s} \sim \pi\right)$, the energy loss is negligible.

We assume that at each point, "thermalization" is quickly established and the distribution is described by the relativistic Maxwell distribution (14). The radiation power integrated within the distance $r$ is

$$
\delta P_{\mathrm{IC}}=4 \pi \iiint r^{2} f\left(r, \Gamma_{W}\right) \frac{d P_{\mathrm{IC}}}{d \Omega_{1}} d \Omega_{1} d r d \Gamma_{W} d E_{\gamma},
$$

where $E_{\gamma}$ is the energy of scattered photons. The normalization $K(r)$ and the averaged Lorentz factor $\Gamma_{0}(r)$ are calculated with the equations of particle conservation and of the energy conservation, that is,

$$
r^{2} \int f(r, \Gamma) d \Gamma=r_{i}^{2} \int f\left(r_{i}, \Gamma\right) d \Gamma
$$

and

$$
m_{e} c^{2} \int \Gamma_{W} f\left(r, \Gamma_{W}\right) d \Gamma_{W}+\frac{\delta P_{\mathrm{IC}}}{4 \pi r^{2} c}=\frac{L_{\mathrm{sd}}}{4 \pi r_{i}^{2} c},
$$

respectively.

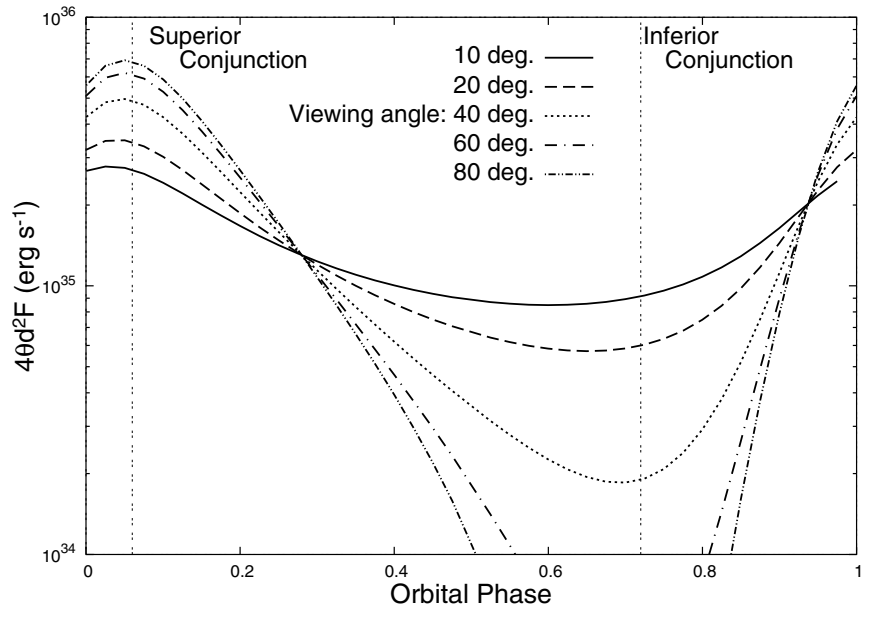

Figure 8. Variation of the integrated flux of the inverse-Compton photons from the cold-relativistic pulsar wind. The results are for a typical Lorentz factor of $\Gamma_{W, 0}=5 \times 10^{3}$. The different lines show the results for the different Earth viewing angles measured from the direction perpendicular to the orbital plane. The phase zero $\phi=0$ corresponds to periastron, and the vertical double-dotted lines indicate the positions of the superior conjunction $(\phi=0.06)$ and the inferior conjunction $(\phi=0.72)$, respectively.

Additionally, we calculated the emissions with monoenergetic distribution and single power-law distribution of the pulsar wind. For the mono-energetic distribution, we found that the shape of the calculated spectra resembles the one calculated with the relativistic Maxwell function. For the power-law distribution, we assumed that the particles are accelerated above $\Gamma_{0}\left(r_{i}\right) \sim 5 \times 10^{3}$ with a power-law index of 3-4, which has been predicted by the plasma simulations of the magnetic reconnection process (Zenitani \& Hoshino 2007). We found that although the IC spectrum extends to very high energy bands, its contribution to the "soft" power-law index 3-4 is much smaller than the shock emissions. Therefore, within the present framework of the particle distributions, the main results discussed in Section 4 are not modified.

Figure 8 summarizes the temporal variations of the integrated flux of IC emissions with respect to the orbital phase. The different curves represent the results for different Earth viewing angles measured from the direction perpendicular to the orbital plane. As we show in Figure 8, the model light curves tend to have a peak around SUPC. This is (1) because the IC photons emitted toward the Earth are produced by the headon-like collision process and (2) because SUPC $(\phi \sim 0.06)$ is close to the periastron $(\phi=0)$, where the separation between two stars becomes minimum and hence the soft photon number density at the location of the pulsar becomes maximum. As a result, the IC process is more efficient around SUPC. Around INFC, the flux becomes minimum since the IC photons traveling toward the Earth are produced by the tail-on collision process. In Figure 8, we show that a larger Earth viewing angle predicts a larger amplitude of the modulation. This is because as the Earth viewing angle approaches edge-on, the amplitude of the variation of the collision angle, which is the angle between the stellar photons and the pulsar wind that emits photons toward the Earth, along the orbital phase increases. We also find a tendency in Figure 8 that as the Earth viewing angle becomes small, the positions of flux maximum and minimum shift toward the periastron $(\phi=0)$ and apastron $(\phi=0.5)$, respectively. This is related to the fact that IC emissivity depends on (1) the collision angle and (2) the number density of the soft photons. 
For a larger Earth viewing angle, the variation of the collision angle with the orbital phase affects the variation of the IC flux. In such a case, the flux maximum (or minimum) appears at the superior conjunction (or inferior conjunction). If the Earth viewing angle approaches zero, the orbital variation of the collision angle is small, and the variation of number density of soft photons at the emission regions mainly causes the variation of the IC flux. In such a case, the IC flux becomes maximum (or minimum) at the periastron (or apastron), where the photon number density at the emission region becomes maximum (or minimum).

\subsection{Shock Emissions}

At the shock, the kinetic energy of the pulsar wind is converted into the internal energy of the wind, and the distribution of particles at the shock is assumed to be described by a power law over several decades in energy. The minimum Lorentz factor $\left(\gamma_{e, \min }\right)$ of the shocked pulsar wind particles is assumed to be the average Lorentz factor of the cold-relativistic pulsar wind at the shock $\left(\Gamma_{0}\left(d_{s}\right)\right.$; see Figure 7$)$. The maximum Lorentz factor $\gamma_{e, \max }$ is determined by balancing between the acceleration timescale $t_{a} \sim \gamma_{e} m_{e} c /(e B)$ and the synchrotron loss timescale $t_{s} \sim 9 m_{e}^{3} c^{5} /\left(4 e^{4} B^{2} \gamma_{e}\right)$. In this paper, we assume $p=2.1$ for the power-law index of the distribution of the particles accelerated at the shock.

Since the ratio of the pulsar wind momentum to the stellar wind momentum is much smaller than unity $(\eta \ll 1)$, we approximate that the flow of the shocked pulsar wind points radially outward from the companion star. In this study, we assume that the velocity $\left(v_{\mathrm{PW}}\right)$ of the bulk motion of shocked pulsar wind does not change with the radial distance, that is, $v_{\mathrm{PW}}(r)=$ constant, because the high-energy emission occurs in the vicinity of the shock.

In the downstream region, the particles lose their energy via the cooling processes. With the steady state approximation, the evolution of the distribution function $N\left(r, \gamma_{e}\right)$ is given by

$$
\frac{\partial N}{\partial r}+\frac{\partial}{\partial \gamma_{e}}\left(\frac{d \gamma_{e}}{d r} N\right)=Q\left(\gamma_{e}\right) \delta\left(r-r_{s}\right),
$$

where $Q\left(\gamma_{e}\right)$ is the source function at the shock. The energy loss of the particles is calculated from

$$
\frac{d \gamma_{e}}{d r}=\frac{1}{v_{\mathrm{PW}}}\left[\left(\frac{d \gamma_{e}}{d t}\right)_{a d}+\left(\frac{d \gamma_{e}}{d t}\right)_{\mathrm{syn}}+\left(\frac{d \gamma_{e}}{d t}\right)_{\mathrm{IC}}\right]
$$

We apply the adiabatic loss given by

$$
\left(\frac{d \gamma_{e}}{d t}\right)_{a d}=\frac{\gamma_{e}}{3 n} \frac{d n}{d t}=-\frac{2 v_{\mathrm{PW}}}{3 r},
$$

where $n$ is the particle number density, and we apply $n r^{2}=$ constant. The synchrotron loss is given as

$$
\left(\frac{d \gamma_{e}}{d t}\right)_{\mathrm{syn}}=-\frac{4 e^{4} B^{2} \gamma_{e}^{2}}{9 m_{e}^{3} c^{5}}
$$

where we used the average pitch angle because we expected that the magnetic field in the shocked pulsar wind is easily randomized. The IC energy loss rate is

$$
\left(\frac{d \gamma_{e}}{d t}\right)_{\mathrm{IC}}=-\iint\left(E-E_{S}\right) \frac{\sigma_{\mathrm{IC}} c}{m_{e} c^{2} E_{s}} \frac{d N_{s}}{d E_{s}} d E_{s} d E,
$$

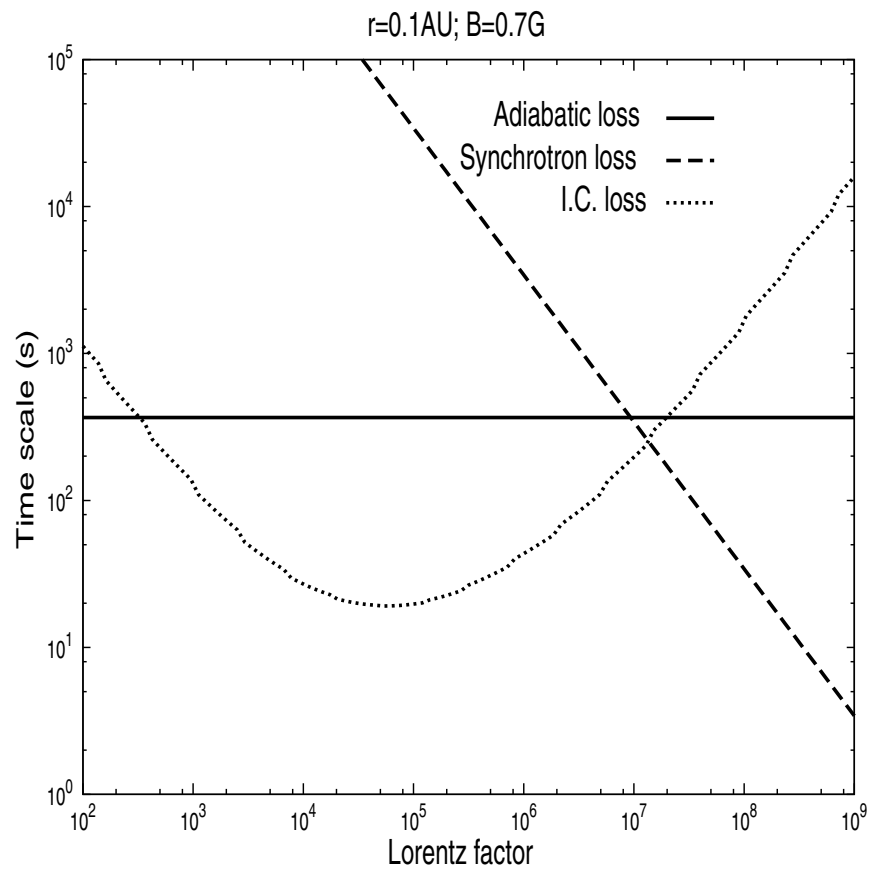

Figure 9. Timescales of the adiabatic loss (solid line), synchrotron loss (dashed line), and IC loss (dotted line). The results are for the radial distance $r=0.1 \mathrm{AU}$ from the pulsar in the opposite direction of the companion star and the magnetic field $B=0.5 \mathrm{G}$. The turnover of the IC loss at the Lorentz factor $\sim 10^{5}$ is due to the Klein-Nishina effect.

where $d N_{s} / d E_{s}$ is the stellar photon field distribution and $\sigma_{\text {IC }}$ is the cross-section for the isotropic photon field. We estimate the magnetic field just behind the shock as

$$
B\left(r_{s}\right)=3\left(\frac{L_{\mathrm{sd}} \sigma\left(r_{s}\right)}{r_{s}^{2} c\left[1+\sigma\left(r_{s}\right)\right]}\right)^{1 / 2},
$$

where $\sigma\left(r_{s}\right)$ is the magnetization parameter at the shock. In the downstream region, we consider the magnetic field to evolve as $B(r) r=$ constant.

We assume that the magnitude of the magnetization parameter at the shock depends on the shock distance from the pulsar because we expect that an energy conversion from the magnetic field to the particle energy of the cold-relativistic pulsar wind gradually decreases the magnetization parameter with the radial distance from the pulsar. Because there is theoretical uncertainty for the evolution of $\sigma$ with the radial distance, we describe the magnetization parameter at the shock with a single power-law function,

$$
\sigma\left(r_{s}\right)=\sigma\left(r_{\text {apex }, 0}\right)\left(\frac{r_{s}}{r_{\text {apex }, 0}}\right)^{-\alpha},
$$

where $r_{\text {apex }, 0}$ is the shock apex distance at the periastron. The evolution of the magnetization parameter for the gamma-ray binary PSR B1259-63/LS 2883 system is also used to explain the X-ray/TeV emissions (Takata \& Taam 2009; Kong et al. 2011, 2012). We argue in Section 5.1 that the $\alpha$ index affects the predicted flux at the TeV energy band.

We expect that the high-energy emission processes occur in the vicinity of the shock surface. Figure 9 shows the timescales of the radiation losses; the solid, dashed, and dotted lines show the timescales of the adiabatic loss, the synchrotron loss, and the IC loss, respectively. The results are for the radial distance $r=0.1 \mathrm{AU}$ from the pulsar in the opposite direction of the companion star and the magnetic field $B \sim 0.5 \mathrm{G}$. 
Since the timescale of the adiabatic loss $\left(\sim 3 r / 2 v_{\mathrm{PW}}\right)$ represents the crossing timescale of the shock region, we show in Figure 9 that the crossing timescale of the particles with a Lorentz factor $\gamma_{e}>10^{3}$ is longer than the timescale of the radiation losses, implying the accelerated particles lose most of their energy in the vicinity of the shock surface through the radiation processes. In the present calculation, therefore, we take into account the emissions occurring between the shock distance $r_{s}$ and the radial distance $3 r_{s}$, beyond which the emissions of the cooled particles are negligible.

Finally, we expect that effect of the Doppler boosting due to the finite velocity of the shocked pulsar wind is the main reason for the temporal variation of the X-ray emissions with the orbital phase. The Doppler boosting introduces an orbital modulation of the emissions that are isotropic in the comoving frame of the flow. Dubus et al. (2010) suggested that the observed orbital modulation of the X-ray emissions from LS 5039 is the result of the Doppler boosting of the shocked pulsar wind with a mildly relativistic speed, $\beta_{\mathrm{PW}} \equiv v_{\mathrm{PW}} / c \sim 0.15-0.3$. Note that this scenario will be different from the case of the gamma-ray binary PSR B1259-63/LS2883. For the PSR B1259-63/LS2883 system, which has a highly eccentric orbit with $e=0.87$, the shock distance from the pulsar varies by about a factor of 10 along the orbital phase. This large variation in the shock distance can produce a large temporal variation of the synchrotron emissions from the shock (Tavani \& Arons 1997; Takata \& Taam 2009; Kong et al. 2011, 2012). With a moderate eccentricity $(e \sim 0.35)$, on the other hand, the shock distance of the LS 5039 system varies by only about a factor of two along the orbital phase. The slight change in the shock distance with the orbital phase will not reproduce the observed temporal variation in X-ray emissions from LS 5039.

\subsection{Pair-creation Process}

The high-energy TeV gamma-rays may be converted into the electron and positron pairs by colliding with the soft photons from the companion star. The mean free path of the pair-creation process of a photon with an energy $E_{\gamma}$ at a radial distance $\tilde{r}$ from the companion star is calculated from

$$
\frac{1}{\ell\left(\tilde{r}, E_{\gamma}\right)}=\left(1-\cos \theta_{\gamma \gamma}\right) \int_{E_{c}}^{\infty} d E_{s} \sigma_{\gamma \gamma} d N_{s} / d E_{s},
$$

where $d N_{s} / d E_{s}$ is the distribution of the number density of the stellar soft photon, and

$$
\sigma_{\gamma \gamma}=\frac{3}{16} \sigma_{T}\left(1-v^{2}\right)\left[\left(3-v^{2}\right) \ln \frac{1+v}{1-v}-2 v\left(2-v^{2}\right)\right]
$$

where $\sigma_{T}$ is the Thomson cross-section, $v\left(E_{\gamma}, E_{s}\right)=$ $\sqrt{1-E_{c} / E_{\gamma}}$, and $E_{c}=2\left(m_{e} c^{2}\right)^{2} /\left[\left(1-\cos \theta_{\gamma \gamma}\right) E_{s}\right]$ with $\theta_{\gamma \gamma}$ being the collision angle.

Since the electron and positron pairs created by $\mathrm{TeV}$ gammarays have a Lorentz factor of $\sim 10^{6}$, they may emit new gamma-rays via the IC process. The gamma-rays emitted by the pairs in turn produce the next generation of pairs. Hence, the $\mathrm{TeV}$ gamma-rays emitted toward the companion star develop the pair-creation cascade process. It has been suggested that the contribution of the emissions from the new generation of the pairs will be important for the emissions around the SUPC phase, where the companion star is located between the pulsar and the observer (e.g., Sierpowska-Bartosik \& Torres 2007; Yamaguchi \& Takahara 2010; Cerutti et al. 2010). In this calculation, we
Table 1

Model Fitting Parameters

\begin{tabular}{lc}
\hline \hline Pulsar & \\
$L_{\text {sd }}$ & $2 \times 10^{36} \mathrm{erg} \mathrm{s}^{-1}$ \\
$P$ & $0.1 \mathrm{~s}$ \\
$B_{s}$ & $2 \times 10^{12} \mathrm{G}$ \\
\hline Pulsar wind & \\
$\Gamma_{0}\left(r_{i}\right)$ & $5 \times 10^{3}$ \\
\hline Shocked pulsar wind & \\
$\gamma_{\text {min }}$ & $\Gamma_{o}\left(r_{s}\right)$ \\
$p$ & 2.1 \\
$\beta_{\mathrm{PW}}$ & 0.15 \\
\hline System & \\
$d$ & $2.5 \mathrm{kpc}$ \\
$i$ & $65^{\circ}$ \\
\hline
\end{tabular}

Notes. From top to bottom, the spin-down power of pulsar $\left(L_{\mathrm{sd}}\right)$, rotation period $(P)$, surface magnetic field $\left(B_{s}\right)$, typical Lorentz factor of the injected coldrelativistic pulsar wind $\left(\Gamma_{o}\right)$, minimum Lorentz factor of the electrons/positrons accelerated at the shock $\left(\gamma_{\min }\right)$, index of the power-law distribution $(p)$, the velocity of the post-shocked flow in units of speed of light $\left(\beta_{\mathrm{PW}}\right)$, distance to the system $(d)$, and inclination angle $i$, respectively.

assume that the created pair travels straight in the direction of the momentum of the incident gamma-rays.

\subsection{Model Parameters}

In Table 1, we summarize the parameters assumed in the model fitting. The observed fluxes are explained by a spin-down power $L_{\text {sd }} \sim 2 \times 10^{36} \mathrm{erg} \mathrm{s}^{-1}$. With $L_{\text {sd }} \sim 2 \times 10^{36} \mathrm{erg} \mathrm{s}^{-1}$, if the pulsar has a typical magnitude of the surface magnetic field, $B_{s} \sim 2 \times 10^{12} \mathrm{G}$, the dipole radiation model of the pulsar spindown predicts the rotation period of $P \sim 0.1 \mathrm{~s}$, implying the gap factional thickness (see Equation (9)) and luminosity (see Equation (8)) of the gap emissions corresponding to $f_{\text {gap }} \sim 0.3$ and $L_{\gamma} \sim 0.03 L_{\mathrm{sd}}$, respectively. Equation (12) implies that the initial Lorentz factor of the kinetically dominated flow is of the order of $\Gamma_{0}=10^{4}$. In the present calculation, we apply $\Gamma_{0}\left(r_{i}\right)=5 \times 10^{3}$ to fit the data. The standard Fermi first-order shock acceleration model has implied that $p \sim 2$ is a typical power-law index of the distribution of the accelerated particles (e.g., Longair 1994 and references therein). The index $p \sim 2$ is also expected from the typical photon index $\alpha_{X} \sim 1.5$ of the observed X-ray emissions from LS 5039. In the fitting, we apply $p \sim 2.1$. We adopt the flow velocity $\beta_{\mathrm{PW}}=0.15$ and the Earth viewing angle $i=65^{\circ}$ to reproduce the amplitudes of the orbital modulation of the observed X-ray and gamma-ray emissions. The distance to the system is assumed to be $2.5 \mathrm{kpc}$.

\section{COMPARISON WITH THE MULTI-WAVELENGTH OBSERVATIONS}

\subsection{GeV Emissions}

The calculated phase-resolved spectra and the light curves are compared with the results of Fermi in Figures 3 and 4, respectively. We show in the figures that the emissions from the outer gap (dashed lines), from cold-relativistic pulsar wind (dotted lines), and from the shocked pulsar wind (dashed-dotted lines) all contribute to the emissions observed by Fermi. The emissions from the cold-relativistic pulsar winds contribute to the emissions around $0.1-0.5 \mathrm{GeV}$ and dominate the other 

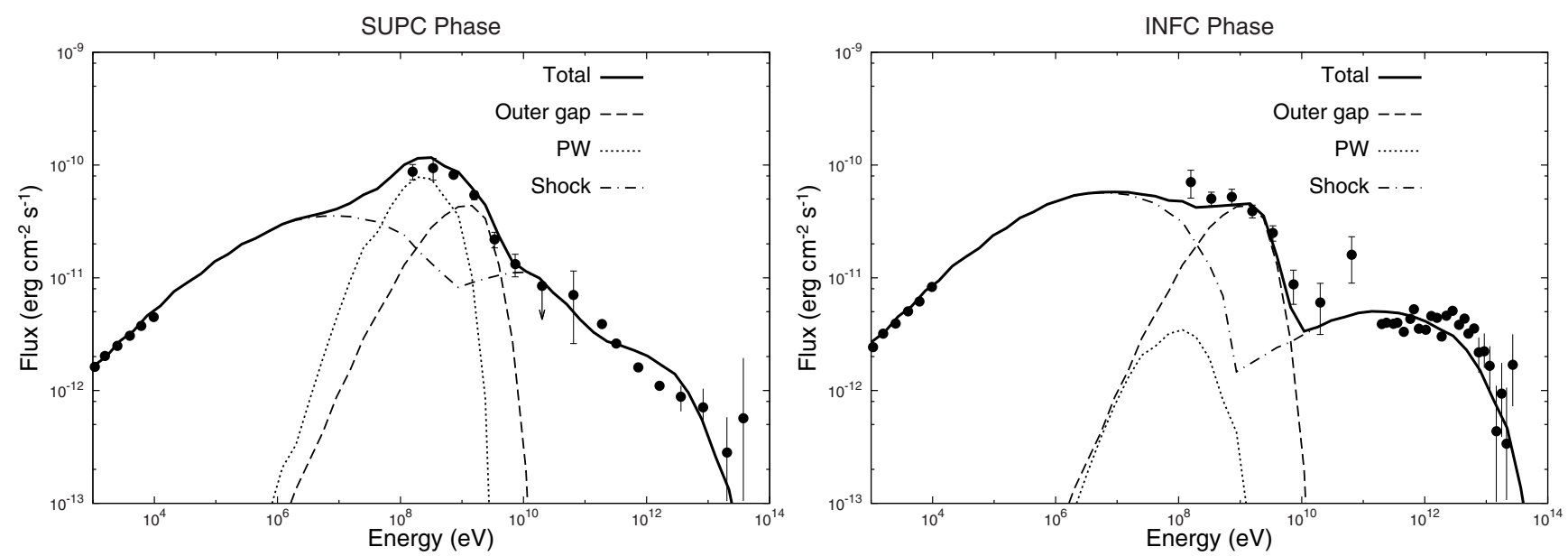

Figure 10. Multi-wavelength spectra of LS 5039 averaged over the SUPC phase, $0.9<\phi<0.45$ (left) and INFC phase, $0.45<\phi<0.9$ (right), respectively. The dashed line, dotted line, and dashed-dotted line represent the calculated spectra of the emissions from the outer gap, cold-relativistic pulsar, and shocked pulsar wind, respectively. The solid lines show the total emissions. The results are for $L_{\mathrm{sd}} \sim 2 \times 10^{36} \mathrm{erg} \mathrm{s}^{-1} \sigma\left(r_{\text {apex }, 0}\right) \sim 0.2, \alpha=2$ and $p=2.1$. The observation data are taken from Takahashi et al. (2009) for X-rays, from the current work for GeV, and from Aharonian et al. (2006) for TeV gamma-rays.

two components around the SUPC. The outer gap emissions dominate the other two components in the $1-10 \mathrm{GeV}$ energy bands for the entire orbit. Above $\sim 10 \mathrm{GeV}$, the emissions are dominated by the IC process of the shocked pulsar wind. We find that the calculated spectra and the light curves for the total emissions (solid lines) in the figures explain major properties of the observations: (1) the $\mathrm{GeV}$ flux becomes maximum around the SUPC and becomes minimum around INFC, (2) the amplitude of the light curve for the $>0.2 \mathrm{GeV}$ bands is larger than that for the $>1 \mathrm{GeV}$, and (3) there is a spectral cutoff at $\sim 2 \mathrm{GeV}$. The present model predicts that the emissions from the cold-relativistic pulsar wind make the spectrum softer around the SUPC (see Figure 12), which is also consistent with the observation. We found that within the framework of the calculations, it would be difficult to explain the position of the upper limit at $\sim 20 \mathrm{GeV}$ for the orbital phase $0.83<\phi<1.17$ (see Section 5.5).

\subsection{Multi-wavelength Emissions}

Figure 10 compares the calculated spectra with the multiwavelength observations. In the figures, the dashed line, dotted line, and dashed-dotted line represent the calculated spectra of the curvature emission in the outer gap, of the IC process of a cold-relativistic pulsar, and of the shock emissions (synchrotron below $\sim 100 \mathrm{MeV}$ and IC above $\sim 100 \mathrm{MeV}$ ), respectively. The solid lines show the spectra combining each component. In the calculation, we assumed that the magnetization parameter at the shock decreases with the inverse square of the shock distance, that is, $\sigma\left(r_{s}\right)=0.2\left(r_{s} / r_{\text {apex }, 0}\right)^{-2}$.

\subsubsection{Shock-I versus Shock-II}

One important prediction in the present scenario is that the $\mathrm{X}$-rays and $\mathrm{TeV}$ gamma-rays originate from different shock regions. We have assumed that there are two kind of shocks, that is, one (Shock-I) is located in a position at which the pulsar wind pressure and stellar wind pressure are in balance, and the other (Shock-II) is located at $r_{s} \sim D_{\text {sep }}$ in the opposite direction of the companion star (see Figure 5). Figure 11 compares the respective contributions of the emissions from Shock-I (solid line) and Shock-II (dashed line) to the total emissions. We find in the figure that the $\mathrm{X}$-ray and $\mathrm{TeV}$ emissions are mainly produced by the Shock-I and Shock-II regions, respectively.

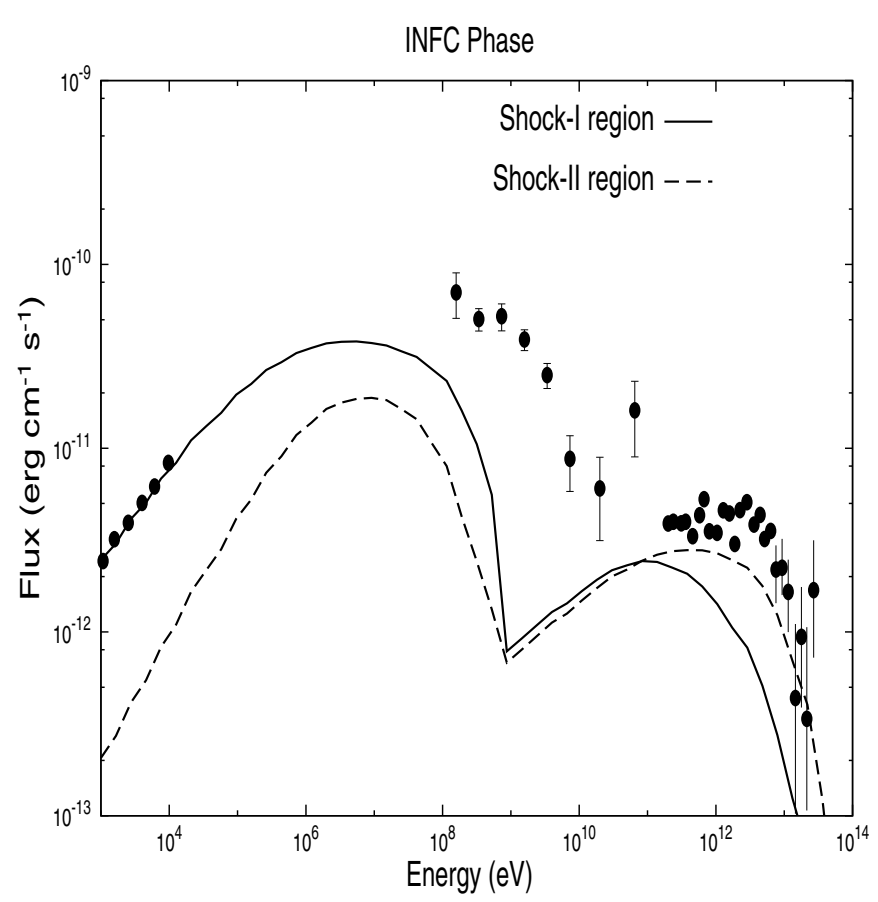

Figure 11. Multi-wavelength spectra of the INFC phase. The solid line and dashed line show the calculated spectra of the particles accelerated at Shock-I region and Shock-II region, respectively. The emissions from the magnetosphere and the cold-relativistic pulsar wind are not displayed in the figure.

The difference in the spectral properties of the Shock-I and Shock-II are caused by the difference in the assumed magnetic field strength at each region. When calculating, we assumed that the magnetization parameter develops as $\sigma\left(r_{s}\right)=$ $0.2\left(r_{s} / r_{\text {apex }, 0}\right)^{-2}$, which produces $B \sim 15 \mathrm{G}$ and $B \sim 0.5 \mathrm{G}$ as the magnetic field strength of the Shock-I and Shock-II, respectively. As we show in Figure 11, the synchrotron radiation of Shock-I is stronger than that of Shock-II. In the Shock-I region, the synchrotron cooling timescale for the particles with a Lorentz factor $\gamma_{e}>10^{6}$ is shorter than the IC cooling timescale, and as a result, the spectrum of IC does not extend beyond $\sim 0.5$ $\mathrm{TeV}$. With $B \sim 0.5 \mathrm{G}$ in the Shock-II region, on the other hand, the IC cooling dominates the synchrotron cooling for the 

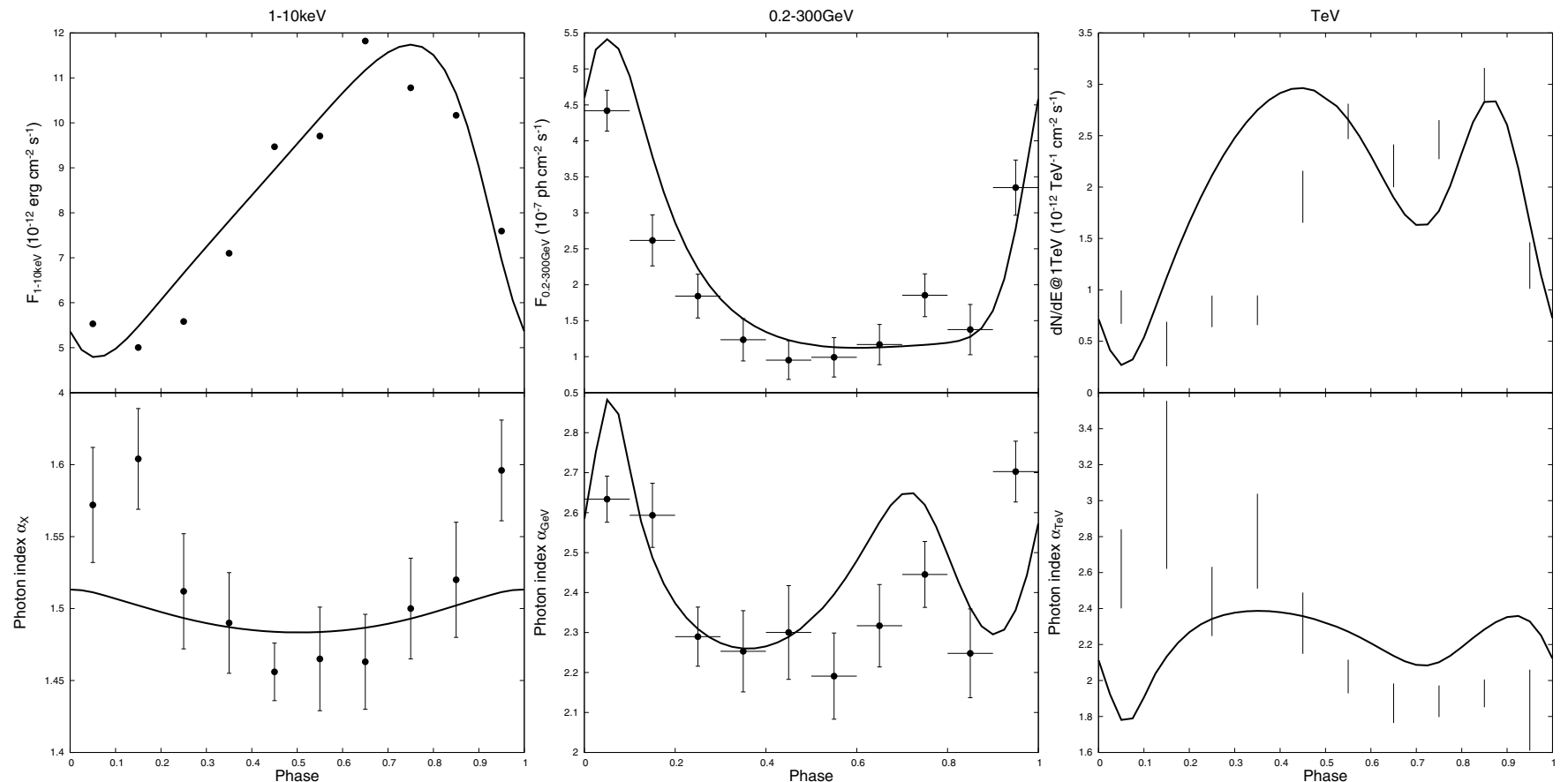

Figure 12. Orbital variation of the flux and photon index in X-ray (left panel), GeV (middle panel), and TeV (right panel), respectively. $\mathrm{X}$-ray data and TeV data were taken from Takahashi et al. (2009) and Aharonian et al. (2006), respectively. The GeV data show the result of the present work (Section 2). The lines show the results of the emission model discussed in Section 3.

electrons/positrons with a Lorentz factor up to $\gamma_{e} \sim 10^{7}$, and hence the spectrum of IC emissions can extend to $\sim 5 \mathrm{TeV}$.

The result that $\mathrm{TeV}$ emissions are produced by the ShockII region with a magnetic field of $\sim 0.5 \mathrm{G}$ is consistent with the results obtained by Zabalza et al. (2013). We showed that $\mathrm{X}$-ray emissions in the Shock-II region could not explain the $\mathrm{X}$-ray observation, which is also consistent with the conclusion of Zabalza et al. (2013). Hence, we propose that the particles accelerated at the Shock-I produces the X-rays via synchrotron radiation, while Zabalza et al. (2013) assumed that the shocked particles produce the Gev emissions via the IC process. As we discussed in Section 4.1, our model expected that the 0.1-10 $\mathrm{GeV}$ emissions are composed of magnetospheric and pulsar wind emissions.

\subsubsection{Orbital Variations}

Figure 12 summarizes the orbital variations of the flux (top panels) and photon index fitted by a single power-law function (bottom panels) for $1-10 \mathrm{keV}$ (left), $0.2-300 \mathrm{GeV}$ (middle), and $0.2-5 \mathrm{TeV}$ (right), respectively. For X-ray emissions (left panel), we find that the Doppler boosting with $\beta_{\mathrm{PW}}=0.15$ of the postshock flow velocity can reproduce the observed amplitude in the $\mathrm{X}$-ray band. This result is consistent with that of Dubus et al. (2010). With $p=2.1$ for the power-law index of the particle distribution at the shock, the predicted photon index $\alpha_{X} \sim 1.5$ is qualitatively consistent with the result of the observation.

For $0.1-1 \mathrm{GeV}$ energy bands, the cold-relativistic pulsar wind contributes to the calculated emissions, and therefore the orbital modulation of the flux shows different behavior from what the X-ray emissions show, as shown in Figure 12. The coldrelativistic pulsar wind mainly produces $0.2-0.5 \mathrm{GeV}$ gammarays, and its emissions dominate the outer gap/shock emissions around SUPC (see Figure 3). Hence, the calculated light curve in the GeV energy band has a flux peak around the SUPC. This model predicts that the GeV spectrum is softer around the SUPC and harder around the apastron. We also show in Figure 12 that the GeV spectrum locally becomes soft around the INFC. This is because the IC process of the shocked pulsar wind is less efficient around the INFC (see the right panel of Figure 12), and as a result, the flux above $10 \mathrm{GeV}$ tends to decrease around the INFC. We show in Figure 12 that the properties of the calculated orbital modulation of $\mathrm{GeV}$ gamma-rays are consistent with the observations.

For TeV energy bands (right panel), we find that the calculated light curve shows a double-peak structure around the INFC, which could be consistent with the observations. Since most of the photons emitted around the SUPC cannot escape from the pair-creation process, the $\mathrm{TeV}$ flux tends to increase as the pulsar moves toward the INFC. As the pulsar approaches to INFC, since the IC process with the tail-on-like collision produces the $\mathrm{TeV}$ photons traveling toward the Earth, the radiation efficiency decreases. In the calculated light curve, therefore, a dip appears around the INFC.

The present model overestimates a $\mathrm{TeV}$ flux at the $0.1-0.5$ orbital phase, as the top right panel in Figure 12 shows. Since the $\mathrm{TeV}$ gamma-rays are mainly produced at the Shock-II region (see Figure 11), this discrepancy may suggest that 3D geometry of the Shock-II region is more complex than one assumed in this study, for which the result of 2D simulation has been applied. The detailed analysis with the shock geometry obtained by 3D simulation will be worth investigating further.

We also find in the bottom panel that difference between the observed and predicted photon indexes in the $0.2-5 \mathrm{TeV}$ energy band is large around SUPC; the calculated spectrum around SUPC becomes very hard compared with the observed spectrum. The predicted hard spectrum at the $0.2-5 \mathrm{TeV}$ energy band is caused by the effect of the pair-creation process. Since the optical depth around $0.1 \mathrm{TeV}$ is larger than that around $1 \mathrm{TeV}$, the pair-creation process absorbs $0.1 \mathrm{TeV}$ photons more than $1 \mathrm{TeV}$ photons, and hence the spectrum at $0.2-5 \mathrm{TeV}$ tends to have a photon index smaller than two. 


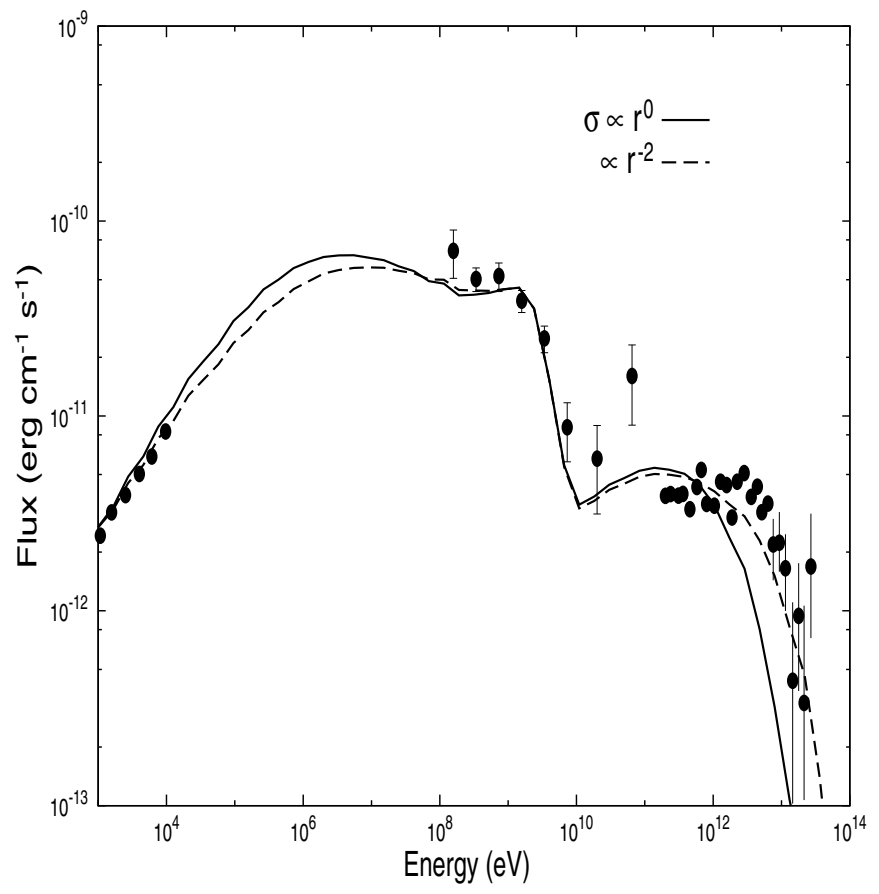

Figure 13. Multi-wavelength spectra of the INFC phase. The different lines show the calculated spectra for different power-index $\alpha$ in the radial distribution of the magnetization parameters $\sigma\left(r_{s}\right) \propto r_{s}^{-\alpha}$. The results are for $\alpha=0$ (solid line) and 2 (dashed line).

The large difference in photon indexes may suggest that an additional component, for example, the nebula component (Bednarek \& Sitarek 2013), contributes to the observed TeV emissions around the SUPC.

\section{DISCUSSION}

\subsection{Dependency on the Magnetization Parameter}

The calculated spectrum in the TeV energy bands, in fact, depends on how the magnetization parameter at the shock evolves with the shock distance from the pulsar, that is, the power-index $\alpha$. Figure 13 compares the calculated spectra of the INFC phase with index $\alpha=0$ (solid line) and 2 (dashed line). As we can see in Figure 13, the calculated spectrum with the constant magnetization parameter $(\alpha=0)$ predicts a cutoff energy in TeV bands much smaller than the observations. Since a smaller index $\alpha$ predicts a larger magnetic field, and hence a larger synchrotron cooling in the Shock-II region, the TeV emissions are suppressed. In the present model, therefore, a larger power index $\alpha$ is preferable for explaining the observed simultaneous $\mathrm{X}$-ray and $\mathrm{TeV}$ emissions.

\subsection{Dependency on the Particle Distribution}

Figure 14 summarizes the dependency of the TeV spectra on the power-law index of the energy distributions of the particles at the shock; the left and right panels show the spectra for the SUPC phase and the INFC phase, respectively. As the SUPC phase, we can see that the calculated spectrum with the index $p=1.9$ (dashed line) will be too hard compared with the observed results, while the calculated spectrum with $p=2.3$ (dotted line) is too soft. Within the framework of the current model, therefore, the index $p \sim 2.1$ (solid line) provides a better fit for the observed TeV spectra of SUPC. We also note that the observed index $\alpha_{X}=1.5 \sim 1.6$ of the X-ray emissions are fitted better by $p \sim 2.1$.

\subsection{Dependency on Parameter $\eta$}

The ratio $(\eta)$ of the momenta of the pulsar wind and the stellar wind is also the model parameter. By assuming $L_{\mathrm{sd}} \sim$ $2 \times 10^{36} \mathrm{erg} \mathrm{s}^{-1}$, which explains the observed flux with $d=2.5$ kpc, and using the typical mass-loss rate $\dot{M} \sim 10^{-7} M_{\odot} \mathrm{yr}^{-1}$ of O-type main-sequence stars, we have applied the ratio $\eta=0.05$ in the present calculation. With the present calculation, it is difficult to constrain the reasonable range of possible $\eta$ by the fitting of the observational results. We have used the momentum ratio to determine the distance to the shock apex from the pulsar. The magnetic field strength at the shock is an important quantity to determine the properties of the calculated spectra. In the present study, however, since the magnetization parameter and hence the magnetic field strength at the shock are also model parameters, we can adjust the magnetic field strength for each $\eta$ to fit the observed spectra. The reasonable range of $\eta$ cannot be constrained by fitting the observed spectra.

A study of the orbital modulation constrains the range of $\eta$ since the geometry of the shock (e.g., opening angle) depends on $\eta$. The calculation with the Doppler effects and 3D geometry of the shock will produce different properties of the orbital modulation for different $\eta$. In the present calculation, however, we ignored the effect of 3D geometry when we calculated the orbital modulation. By assuming that the flow of the shocked pulsar wind points radially outward from the companion star, we took into account only the effect of the Doppler boosting. In such a case, we cannot reasonably constrain the possible $\eta$ by fitting the orbital modulation. The full calculation with $3 \mathrm{D}$ geometry will be subject to future study.

\subsection{Effect of the Pair-creation Cascade}

Figure 15 shows the $\mathrm{GeV} / \mathrm{TeV}$ spectrum of the SUPC phase and compares the calculated spectra with different types of consideration on the pair-creation process. In the left panel, the solid line represents the spectrum including the effects of the pair-creation cascade. The dotted line takes into account the absorption by the pair-creation process, but it ignores the emissions from the created pairs. The dashed line shows the spectrum ignoring the pair-creation process. For LS 5039, because the surface temperature of the companion star is $k T \sim 3 \mathrm{eV}$, the gamma-rays with an energy $0.05-5 \mathrm{TeV}$ are subject to the pair-creation process, as Figure 15 shows. The emissions from the new pairs and the subsequent pair-creation cascade processes affect the spectra at the $\sim 10 \mathrm{GeV}$ energy band, as the dotted line of Figure 15 shows.

In the right panel, the different lines show the calculated $\mathrm{TeV}$ spectra with different optical depths of the pair-creation. The solid line shows the result for the optical depth that assumes $k T_{s}=3.4 \mathrm{keV}$ and the spherically symmetric stellar photon field. There will be several uncertainties related to the stellar photon field at the emission region: (1) the stellar photons field could depend on the latitude (Negueruela et al. 2011); (2) the spectrum would not be exactly described by the Planck function; and (3) the photon density at the emission region will depend the complex shock structure. To see the dependency of the spectral shape in $\mathrm{TeV}$ energy bands, we artificially increased or decreased the optical depth of the pair-creation process. For the dashed line in the right panel of Figure 15, we increased the optical depth by a factor of two, while for the dotted line, we decreased it by a factor of two. As we can see in the figure, the difference in the optical depth affects to the spectrum in $0.1-1$ $\mathrm{TeV}$ energy bands. 

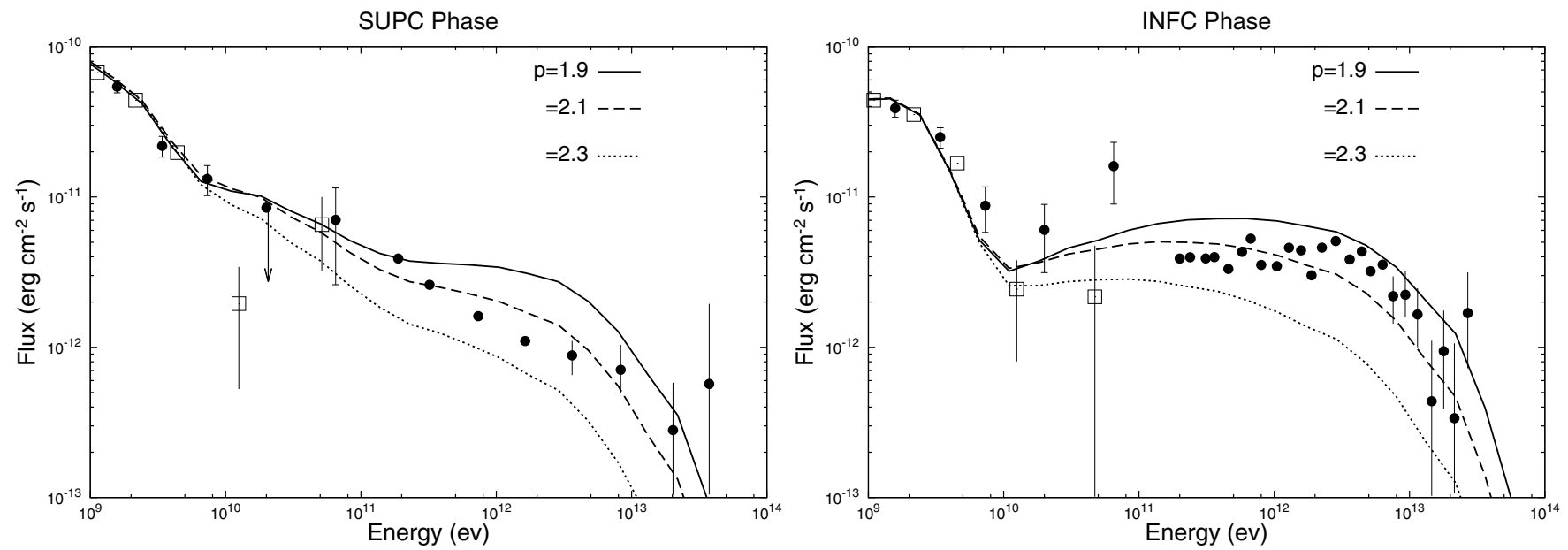

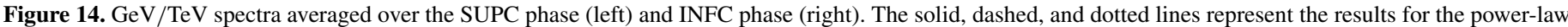
index of $p=1.9,2.1$, and 2.3, respectively. The boxes show the data taken from Hadasch et al. (2012).
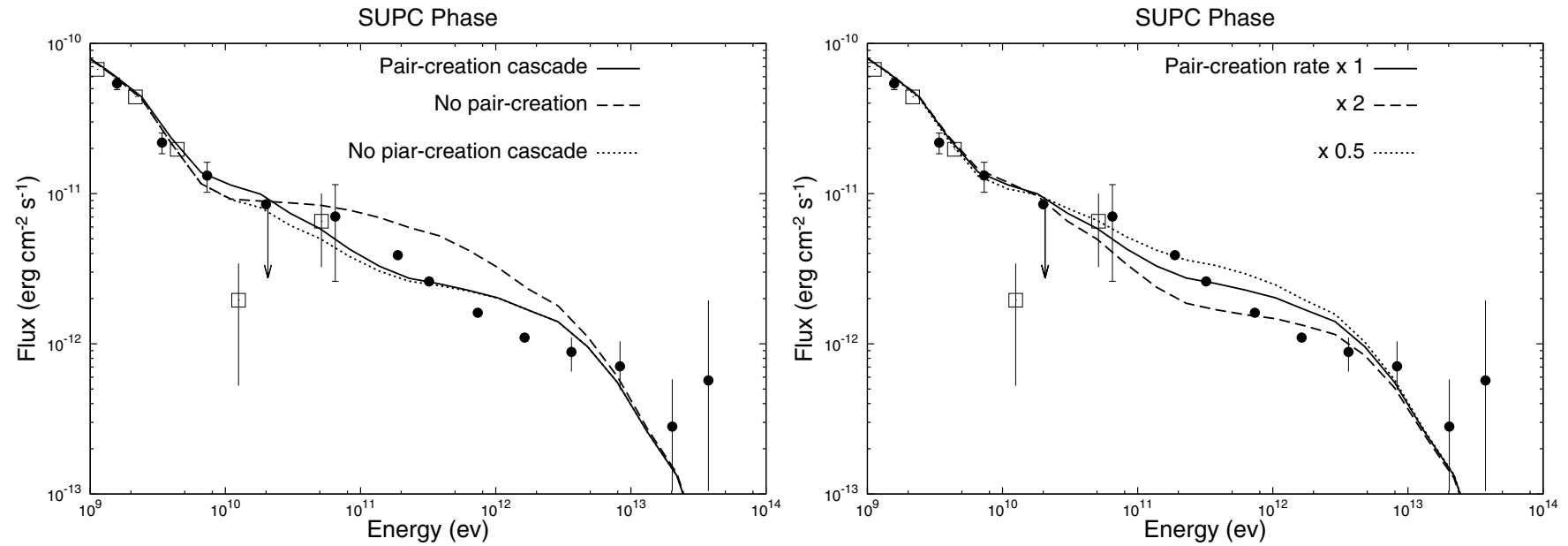

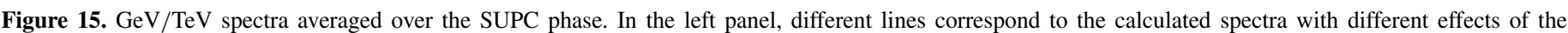

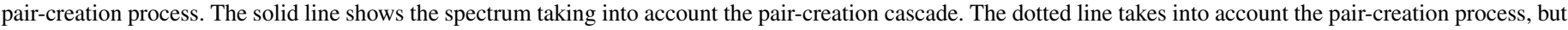

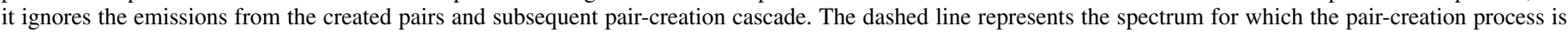

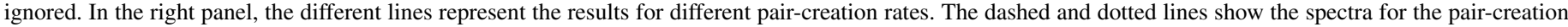
rate increased and decreased by a factor of two, respectively. The boxes show the data taken from Hadasch et al. (2012).

\subsection{0-100 GeV Emissions}

Although the present model can explain many observational properties in the multi-wavelength bands, we are not certain that the present model is consistent with the spectral behavior of the $10-100 \mathrm{GeV}$ emissions at the SUPC phase observed by Fermi. As Figure 14 shows, the calculated spectrum in SUPC does not show a spectral break in $10-100 \mathrm{GeV}$ bands, while the upper limit around $\sim 20 \mathrm{GeV}$ determined by Fermi may suggest the existence of a spectral break. To explain the position of the upper limit, one may consider that the minimum Lorentz factor of the shocked particles is of the order of $\gamma_{e, \min } \sim 10^{5}$. In the present model, since we expected $\Gamma_{0} \sim 5 \times 10^{3}$ for the typical Lorentz factor of the cold-relativistic pulsar wind, we assumed that the shocked particles have a Lorentz factor larger than $\gamma_{e, \text { min }} \sim 5 \times 10^{3}$. Furthermore, we can see that if the minimum Lorentz factor of the shocked particles is larger than $\gamma_{e, \min } \sim 10^{5}$, the predicted spectrum of X-ray emissions becomes much harder than results of the observation, which shows a photon index $\alpha_{X} \sim 1.5$. This hard spectrum in the $0.1-10 \mathrm{keV}$ bands is expected because the energy of the synchrotron photons emitted by the particles with a Lorentz factor $\gamma_{e \text {,min }} \sim 10^{5}$ is larger than $10 \mathrm{keV}$. To investigate the behaviors of emissions in the $10-100 \mathrm{GeV}$ bands of the SUPC, more detailed theoretical and observational studies are required.

\section{SUMMARY}

In this paper, we have discussed the mechanisms of the highenergy emissions from the gamma-ray binary LS 5039. In the first part, we reported on results of the observational analysis using $4 \mathrm{yr}$ data of Fermi and updated the information of the $\mathrm{GeV}$ emissions from LS 5039. We showed that due to the improvement of instrumental response function and increase of the statistics, the flux in $\sim 100 \mathrm{MeV}$ bands has noticeably decreased and uncertainties of the spectra beyond $\sim 10 \mathrm{GeV}$ have been significantly improved. We divided the observation time into three equally spaced orbital phase bins, for which one bin includes the emissions from the superior conjunction. We showed that the spectra of two orbital bins, excluding the superior conjunction, have a clear spectral cutoff at several $\mathrm{GeV}$, and they resemble those of the gamma-ray pulsars. For the bin including the superior conjunction, an enhancement at $0.1-0.3 \mathrm{GeV}$ is seen exclusively, and the spectrum below 10 $\mathrm{GeV}$ is significantly softer compared with the spectra of the other two orbital bins, suggesting an additional component below 1 
$\mathrm{GeV}$. Our results suggest that the $0.1-100 \mathrm{GeV}$ emissions from LS 5039 contain three different components, that is, (1) the first component contributing to $<1 \mathrm{GeV}$ emissions around the superior conjunction, (2) the second component dominating in the $1-10 \mathrm{GeV}$ emissions for the entire component, and (3) the third component, which is compatible with the lower-energy tail of the $\mathrm{TeV}$ emissions

In the second part, we discussed the emission mechanisms of $\mathrm{X}$-ray, $\mathrm{GeV}$, and $\mathrm{TeV}$ gamma-rays. We developed the model in which the curvature emissions from the magnetospheric outer gap and the IC process of the cold-relativistic pulsar wind contribute to the observed $\mathrm{GeV}$ emissions. Our model predicts that the outer gap emissions mainly produce the observed emissions in 1-10 GeV bands for the entire orbit and the observed emissions near $1 \mathrm{GeV}$ are pulsed. The IC process of the cold-relativistic pulsar wind produces the gamma-rays with $0.1-0.5 \mathrm{GeV}$ and contributes to the observed spectrum at the SUPC phase. We applied the shock geometry which resembles that in Zabalza et al. (2013), that is, there are two kinds of termination shock around the pulsar: Shock-I due to the pulsar wind/stellar wind interaction and Shock-II caused by the effect of the orbital motion. We proposed that TeV gammarays are produced via the IC process of the Shock-II region, where the magnetic field strength is $\sim 0.5 \mathrm{G}$. This result in the emission region of the $\mathrm{TeV}$ gamma-rays is consistent with the result obtained by Zabalza et al. (2013). However, our model expects that the particles accelerated at the Shock-I produce the X-rays via synchrotron radiation, while Zabalza et al. (2013) assumed that a strong radiative loss limits the acceleration at Shock-I and the shocked particles produce the Gev emissions via the IC process.

We appreciate the anonymous referee for useful comments and suggestions. J.T. and K.S.C. are supported by a GRF grant of the HK Government under HKU7009 11P. A.K.H.K. is supported by the National Science Council of the Republic of China (Taiwan) through grants NSC100-2628-M-007-002-MY3 and NSC100-2923-M-007-001-MY3. P.H.T. is supported by the National Science Council of the Republic of China (Taiwan) through grant NSC101-2112-M-007-022-MY3. C.Y.H. is supported by the National Research Foundation of Korea through grant 2011-0023383. J.T. thanks the Theoretical Institute for Advanced Research in Astrophysics (TIARA) operated under the Academia Sinica Institute of Astronomy and Astrophysics, Taiwan, which enabled J.T. to use the PC cluster at TIARA.

\section{REFERENCES}

Abdo, A. A., Ackermann, M., Ajello, M., et al. 2009, ApJL, 706, L56 Abdo, A. A., Ackermann, M., Ajello, M., et al. 2011, ApJL, 736, L11
Ackermann, M., Ajello, M., Ballet, J., et al. 2012, Sci, 335, 189

Ackermann, M., Ajello, M., Ballet, J., et al. 2013a, ApJL, 773, L35

Ackermann, M., Ajello, M., Allafort, A., et al. 2013b, ApJS, 209, 34

Aharonian, F., Akhperjanian, A. G., Aye, K.-M., et al. 2005, A\&A, 442, 1

Aharonian, F., Akhperjanian, A. G., Bazer-Bachi, A. R., et al. 2006, A\&A, 460, 743

Albert, J., Aliu, E., Anderhub, H., et al. 2006, Sci, 312, 1771

Aragona, C., McSwain, M. V., Grundstrom, E. D., et al. 2009, ApJ, 698, 514

Ball, L., \& Kirk, J. G. 2000, APh, 12, 335

Bednarek, W., \& Sitarek, J. 2013, MNRAS, 430, 2951

Bosch-Ramon, V., Barkov, M. V., Khangulyan, D., \& Perucho, M. 2012, A\&A, 544, 59

Bregeon, J., Charles, E., \& Wood, M. 2013, in Proc. 2012 Fermi Symposium, eConf C121028, eprint arXiv: 1304.5456

Canto, J., Raga, A. C., \& Wilkin, F. P. 1996, ApJ, 469, 729

Casares, J., Ribó, M., Ribas, I., \& Paredes, J. M. 2005, MNRAS, 360, 1105

Cerutti, B., Malzac, J., Dubus, G., \& Henri, G. 2010, A\&A, 519, 81

Cheng, K. S., Ho, C., \& Ruderman, M. 1986, ApJ, 300, 500

de Jager, O. C. 2007, ApJ, 658, 1177

de Jager, O. C., Harding, A. K., Michelson, P. F., et al. 1996, ApJ, 457, 253

Dubus, G. 2013, A\&ARv, 21, 64

Dubus, G., Cerutti, B., \& Henri, G. 2010, A\&A, 516, 18

Eichler, D., \& Usov, V. 1993, ApJ, 402, 271

Hadasch, D., Torres, D. F., Tanaka, T., et al. 2012, ApJ, 749, 54

Harding, A. K., \& Muslimov, A. G. 2011, ApJL, 726, L10

Hinton, Skilton, J. L., Funk, S., et al. 2009, ApJL, 690, L101

Kapala, M., Bulik, T., Rudak, B., Dubus, G., \& Lyczek, M. 2010, in Proceedings of the 25th Texas Symposium on Relativistic Astrophysics, ed. F. M. Rieger, C. vanEldik, \& Werner Hofmann, PoS (Texas 2010) 193

Khangulyan, D., Aharonian, F. A., Bogovalov, S. V., \& Ribó, M. 2011, ApJL, 742, L98

Khangulyan, D., Aharonian, F. A., Bogovalov, S. V., \& Ribó, M. 2012, ApJL, 752, L17

Kong, S. W., Cheng, K. S., \& Huang, Y. F. 2012, ApJ, 753, 127

Kong, S. W., Yu, Y. W., Huang, Y. F., \& Cheng, K. S. 2011, MNRAS, 416, 1067

Longair, M. S. 1994, in High Energy Astrophysics, Vol. 2, (2nd ed.; Cambridge: Cambridge Univ. Press), 357

Moldón, J., Ribó, M., \& Paredes, J. M. 2012, A\&A, 548, 103

Negueruela, Ignacio, Ribó, M., Herrero, A., Lorenzo, J., Khangulyan, D., \& Aharonian, F. A. 2011, ApJL, 732, L11

Nolan, P. L., Abdo, A. A., Ackermann, M., et al. 2012, ApJS, 199, 31

Ray, P. S., Kerr, M., Parent, D., et al. 2011, ApJS, 194, 17

Sarty, G. E., Szalai, T., Kiss, L. L., et al. 2011, MNRAS, 411, 1293

Sierpowska-Bartosik, A., \& Torres, D. F. 2007, ApJL, 671, L145

Sierpowska-Bartosik, A., \& Torres, D. F. 2008, APh, 30, 239

Takahashi, T., Kishishita, T., Uchiyama, Y., et al. 2009, ApJ, 697, 592

Takata, J., \& Taam, R. E. 2009, ApJ, 702, 100

Takata, J., Wang, Y., \& Cheng, K. S. 2010, ApJ, 715, 1318

Tam, P. H. T., Huang, R. H. H., Takata, J., et al. 2011, ApJL, 736, L10

Tavani, M., \& Arons, J. 1997, ApJ, 477, 439

Torres, D. F. 2011, in Proceedings of the 1st Sant Cugat Forum on Astrophysics, ICREA Workshop on the High-energy Emission from Pulsars and Their Systems, held in 2010 April, preprint, arXiv:1008.0483

Wang, Y., Takata, J., \& Cheng, K. S. 2010, ApJ, 720, 178

Yamaguchi, M. S., \& Takahara, F. 2010, ApJ, 717, 85

Yamaguchi, M. S., \& Takahara, F. 2012, ApJ, 761, 146

Zabalza, V., Bosch-Ramon, V., Aharonian, F., \& Khangulyan, D. 2013, A\&A, 551,17

Zenitani, S., \& Hoshino, M. 2007, ApJ, 670, 702 